ACCEPTED By ApJ

Preprint typeset using $\mathrm{LAT}_{\mathrm{E}} \mathrm{X}$ style emulateapj v. 11/10/09

\title{
SELF-SIMILAR DYNAMICAL RELAXATION OF DARK MATTER HALOS IN AN EXPANDING UNIVERSE
}

\author{
A. Lapi $^{1,2}$ And A. Cavaliere ${ }^{1}$ \\ Accepted by ApJ
}

\begin{abstract}
We investigate the structure of cold dark matter halos using advanced models of spherical collapse and accretion in an expanding Universe. These base on solving time-dependent equations for the moments of the phase-space distribution function in the fluid approximation; our approach includes non-radial random motions, and most importantly, an advanced treatment of both dynamical relaxation effects that takes place in the infalling matter: phase-mixing associated to shell crossing, and collective collisions related to physical clumpiness. We find self-similar solutions for the sphericallyaveraged profiles of mass density $\rho(r)$, pseudo phase-space density $Q(r)$ and anisotropy parameter $\beta(r)$. These profiles agree with the outcomes of state-of-the-art $N$-body simulations in the radial range currently probed by the latter; at smaller radii, we provide specific predictions. In the perspective provided by our self-similar solutions we link the halo structure to its two-stage growth history, and propose the following picture. During the early fast collapse of the inner region dominated by a few merging clumps, efficient dynamical relaxation plays a key role in producing a closely universal mass density and pseudo phase-space density profiles; in particular, these are found to depend only weakly on the detailed shape of the initial perturbation and the related collapse times. The subsequent inside-out growth of the outer regions feeds on the slow accretion of many small clumps and diffuse matter; thus the outskirts are only mildly affected by dynamical relaxation but are more sensitive to asymmetries and cosmological variance.
\end{abstract}

Subject headings: dark matter — galaxies: halos — method: analytical.

\section{INTRODUCTION}

The cosmogonical paradigm envisages galaxies and galaxy systems to form and shine when baryons accrete, settle and condense under the gravitational pull provided by the dark matter (DM), which is cold and collisionless at the binary level. Understanding the formation history and the detailed structure of the DM gravitational wells is needed to provide a firm foundation for an accurate theory of galaxy formation and evolution.

The story starts with DM primordial density perturbations of the cosmic density field; these at first grow by gravitational instability, and as the local gravity prevails are enforced to collapse and virialize into equilibrium 'halos'. The resulting halo growth is hierarchical in mass and sequential in time, with small clumps forming first and then stochastically merging together into larger and more massive objects.

However, describing and understanding the details of the halo formation process has proven to be a complex and tricky task, that still challenges the intense efforts spent by the astrophysical community over the last thirty years. Major empirical improvements occurred with the advent of the technology allowing to run intensive $N$-body simulations of many million to a few billion particles on powerful supercomputers (for a review, see Springel et al. 2005). A number of hot issues are recalled next.

First, DM halos form in two stages (Zhao et al. 2003; Diemand et al. 2007; Stadel et al. 2009; Fakhouri, Ma \& Boylan-Kolchin 2010; Genel et al. 2010; Wang et al.

\footnotetext{
${ }^{1}$ Dip. Fisica, Univ. 'Tor Vergata', Via Ricerca Scientifica 1, 00133 Roma, Italy.

${ }^{2}$ SISSA, Via Bonomea 265, 34136 Trieste, Italy.
}

2011): an early fast collapse of the halo bulk including a few major merger events, which reshuffle the gravitational potential and cause the DM to undergo (incomplete) dynamical relaxation; a late slow growth of the halo outskirts in the form of many minor mergers and diffuse accretion, which little affect the inner potential well, but contribute most (up to $80 \%$ ) of the final mass.

Second, DM halos in equilibrium show an approximately universal spherically-averaged mass distribution. Originally, this was described with the simple formula proposed by NFW (Navarro, Frenk \& White 1997), where the logarithmic density slope $\gamma \equiv-\mathrm{d} \log \rho / \mathrm{d} \log r$ is given by $\gamma=(1+3 \hat{r}) /(1+\hat{r})$ in terms of the radius $\hat{r} \equiv r / r_{-2}$ normalized to the position $r_{-2}$ where $\gamma=2$. This implies that the density profile $\rho(r) \propto \hat{r}^{-1}(1+\hat{r})^{-2}$ features an inner powerlaw behavior $r^{-1}$, steepens outwards to $r^{-2}$ in the halo middle, and then goes into an asymptotic shape $r^{-3}$ in the outskirts. Remarkably, the NFW formula was found to be approximately scaleinvariant, i.e., to fit accurately the simulation outcomes for halos of different mass scales. On the other hand, a weak scale dependence is introduced by the concentration parameter $c \equiv R_{200} / r_{-2}$, that is the ratio between $r_{-2}$ and the radius $R_{200}$ where the overdensity relative to the background amounts to 200; despite the name, it actually constitutes a measure of the halo outer extension. Simulations show that $c \approx 3.5$ applies at the end of the fast collapse (with minor mass and redshift dependencies, see Prada et al. 2011), and increases as $c \propto H(z) \propto(1+z)^{-1}$ afterwards. At the present time $z \approx 0$, a relation $c \propto M^{-0.13}$ applies, with a scatter around 0.2 dex due to variance in the growth histories (see Bullock et al. 2001); typically, a galaxy halo with current mass $M \approx$ a few $10^{12} M_{\odot}$ collapsed at $z \approx 2$ 
features a concentration value $c \approx 10$, while the halo of a galaxy cluster with $M \approx 10^{15} M_{\odot}$ collapsed at $z \approx 0.5$ features a value $c \approx 5$.

In addition, recent simulations (Navarro et al. 2004, 2010) have shown evidence of small but systematic deviations from the simple NFW expression; they rather favor a Sérsic-Einasto (Sérsic 1963, Einasto 1965; see also Prugniel \& Simien 1997; Merritt et al. 2006; Lapi \& Cavaliere 2011) formula $\gamma=\tau+(2-\tau) \hat{r}^{\eta}$, depending on the two parameters $\tau$ and $\eta$ that describe the inner behavior and the curvature of the density profile $\rho(r) \propto r^{-\tau} e^{-(2-\tau) r^{\eta} / \eta}$ before a final exponential cutoff. Current simulations set the upper limit $\tau \lesssim 0.9$ on the inner asymptotic powerlaw but still lack enough resolution to pinpoint the true value. On the other hand, if a pure Einasto (1965) model with $\tau=0$ is adopted, different values of $\eta \approx 0.1-0.2$ are required to provide precise fits to different simulated halos. This indicates that scale-invariance in the mass distribution is actually broken, and/or that a halo's development is appreciably affected by variance related to detailed merging histories or environmental conditions.

Third, DM halos show a definite spherically-averaged profile of anisotropy. This is described on using the standard Binney (1978) parameter $\beta(r) \equiv 1-\sigma_{\theta}^{2}(r) / \sigma_{r}^{2}(r)$ in terms of the radial and tangential velocity dispersions $\sigma_{r, \theta}^{2}(r)$. Halos are found to be quasi-isotropic at the center with $\beta \approx 0$ within the resolution limits, and to become radially anisotropic outwards. Specifically, $\beta \approx 0.25$ applies at $r \approx r_{-2}$ and $\beta \approx 0.5$ at $r \approx$ a few $r_{-2}$; then $\beta$ decreases outwards, though with wide oscillations (e.g., Navarro et al. 2010; Ludlow et al. 2010). Remarkably, within $r_{-2}$ the anisotropy parameter and the slope of the density profile appear to be correlated (see Huss, Jain \& Steinmetz 1999; Hansen \& Moore 2006) through a $\beta-\gamma$ relation of approximate form $\beta(r) \approx-0.15+0.2 \gamma(r)$.

Finally, DM halos show a powerlaw sphericallyaveraged profile of the quantity $Q(r) \equiv \rho / \sigma^{3}$. This has the dimensions of a phase-space density, but it is not a true measure neither of it nor of its coarse-grained version (see discussion by Ascasibar \& Binney 2005; Sharma \& Steinmetz 2006); thus it is often referred to as a pseudo (or a proxy of the) phase-space density, although it is still debated whether the radial or the total velocity dispersion should enter its definition (see discussion by Schmidt, Hansen \& Macció 2008). Remarkably, although the density $\rho(r)$ and velocity dispersion $\sigma^{2}(r)$ have articulated runs, $Q(r)$ follows a simple powerlaw $Q(r) \propto r^{-\chi}$ over three order of magnitude in radius, with the same exponent $\chi \approx 1.9$ applying for all halos (Taylor \& Navarro 2001; Hoffman et al. 2007; Ascasibar \& Gottlöber 2008; Vass et al. 2009; Navarro et al. 2010; Ludlow et al. 2010). On the other hand, the recent simulations highlight that such a powerlaw behavior holds within $r_{-2}$, but that $Q(r)$ possibly steepens in the inner regions, while it flattens appreciably in the outskirts. The origin of the powerlaw behavior is presently unknown, but numerical experiments with different perturbation spectra indicate that it is not related to initial conditions or hierarchical merging (see Wang \& White 2009)

Unfortunately, these remarkable findings still lack a firm theoretical background, while their reliability is restricted in the inner and outer regions of the halos by limited resolution and small particle statistics, respectively. These are good reasons to complement the numerical approach with analytic models.

In the analytic vein, a simple approach is provided by the self-gravitating, static equilibria of DM based on the Jeans equation (Taylor \& Navarro 2001; Austin et al. 2005; Dehnen \& McLaughlin 2005; Lapi \& Cavaliere 2009a, 2009b, 2011)

$$
\frac{1}{\rho} \frac{\mathrm{d}}{\mathrm{d} r}\left(\rho \sigma_{r}^{2}\right)+2 \beta \frac{\sigma_{r}^{2}}{r}+\frac{G M(<r)}{r^{2}}=0 .
$$

Two ingredients are needed to solve it for $\rho(r)$ : the profile of the anisotropy parameter $\beta(r)$, and that of the phasespace density $Q(r)$ linking $\rho(r)$ and $\sigma_{r}^{2}(r)$ in the spirit of an 'equation of state'. Both runs can be predicted from simple scaling arguments, and refined by comparison with numerical simulations (see above), to the effect that $\beta(r)$ takes the form of the linear $\beta-\gamma$ relation, while $Q(r) \propto r^{-1.9}$ applies. Interestingly, the resulting density profiles are well described by the Sérsic-Einasto formula (Lapi \& Cavaliere 2011), and turn out to be very close to the simulation results in the halo middle where the Jeans solutions are reliable; conversely, Ludlow et al. (2011) take up from simulations an Einasto shape for $\rho(r)$ and solve Jeans to closely recover the powerlaw run of $Q(r)$. However, such analytic approaches are limited being based on a static equation; they provide final equilibrium pictures of halos, but little can tell on how DM particles progressively collapse or accrete, and then relax. As such, they sidestep origins and building up of the profiles $Q(r)$ and $\beta(r)$, and are expected to fail close to the halo outskirts, in the region exposed to infall where equilibrium is not yet achieved.

To go beyond these limitations requires formulating physical models of infall and accretion in an expanding universe; to that purpose we resort to an advanced fluid-like description of the DM dynamics including random non-radial motions and relaxation effects, but still amenable to a self-similar treatment. The latter provides a tractable, analytic way of investigating timedependent problems in complex physical systems; it is possible whenever the system dynamics can be characterized, besides spacetime variables, by a few parameters with independent dimensions. Although providing only a particular analytic solution to the physical problem, it often accurately yields the long-time behaviors and offers a useful guide for understanding the generic features of the system (see discussions by Sedov 1959; Zeldovich \& Raizer 1967). In the present context, a self-similar description for the collapse and condensation of a DM halo is allowed by choosing a scale-invariant shape of the initial mass perturbation, and adopting an Einstein-deSitter cosmological framework.

Self-similar solutions for the purely radial infall of collisionless matter have been pioneered by Gunn \& Gott (1972), then explicitly derived by Fillmore \& Goldreich (1984) and Bertschinger (1985) on adopting a Lagrangian description for the orbits of individual particles, and by Teyssier et al. (1997) and Subramanian (2000) on adopting an equivalent fluid approximation for the ensemble of particles. Such self-similar treatments with purely radial 
infall concur in yielding very steep inner density profiles with $\gamma \sim 2$, at variance with the simulation outcomes.

White \& Zaritsky (1991), Ryden (1993), Sikivie et al. (1997), Subramanian (2000), Nusser (2001), Zukin \& Bertschinger (2010a, 2010b), Vogelsberger et al. (2011), and Lithwick \& Dalal (2011) took steps toward fixing the problem by inclusion of non-radial motions originated by tidal torques, either in spherical or triaxial collapses; when angular momentum is present, the system develops a tangential velocity dispersion that causes a flattening of the inner density profile (see also Eq. 1). However, the quantitative effect is sensitive to the specific amount of angular momentum endowed at, or acquired during the infall, so the solutions still fail to explain the approximately universal shapes found in simulations (see discussion by $\mathrm{Lu}$ et al. 2006).

The view we submit here envisages that all such shortcomings go back to a key ingredient missing (or at least, inadequately treated) in the classic self-similar models, i.e., dynamical relaxation. This involves two different mechanisms leading to broadly similar macroscopic outcomes: phase-mixing, a localized process related to spreading of neighboring particle orbits over phase-space; and violent relaxation, a volume process driven by irregular fluctuations of the gravitational potential. Both these effects have long been recognized to play a crucial role in the approach of a gravitational system to its equilibrium configuration, with violent relaxation especially relevant when the initial condition of the collapse is clumpy (e.g., van Albada 1982; Ma \& Bertschinger 2004; $\mathrm{Lu}$ et al. 2006). This is certainly the case in the standard cosmological framework, where DM objects form from accretion punctuated by mergers.

For example, Lynden-Bell (1967) and Nakamura (2000) based on a statistical mechanical approach to show that an equilibrium state may be achieved when violent, full relaxation erases the memory of the initial conditions. Besides delicate issues concerning the mathematical consistency of these theories (see discussion by Arad \& Lynden-Bell 2005), a number of numerical experiments have demonstrated that the relaxation is always incomplete (see van Albada 1982; Henriksen \& Widrow 1999; Trenti, Bertin \& van Albada 2005, and references therein), with a significant correlation retained between the final and initial state of a particle. In fact, this may explain why the detailed density profiles of virialized DM halos ultimately do depend on the formation history and on cosmogonical conditions.

In this paper we propose an advanced treatment of both dynamical relaxation effects within self-similar solutions for the spherical collapse of DM halos in a fluid-like approach. We will show that our description resolves the discrepancy between the outcomes of self-similar models and numerical simulations, in that the solutions of the former feature profiles of mass density, pseudo phasespace density and anisotropy consistent with the latter.

The plan of the paper is straightforward. In $\S 2$ we derive the evolution equations for the moments of the DM distribution function in the fluid approximation, including the terms responsible for dynamical relaxation (see Appendix). In $\S 3$ we reduce these equations to a self-similar form. In $\S 4$ we derive conservation laws, investigate the inner asymptotic behaviors of the solutions, present the full solutions, and compare them with the simulation outcomes. Finally, in $\S 5$ we discuss our approach and summarize our main conclusions.

\section{DM DYNAMICS IN THE FLUID APPROXIMATION}

We consider a spherically symmetric, nonrotating DM halo, and describe particle positions and velocities in terms of the standard polar coordinates $r, \theta, \phi$, and of the corresponding velocity components $v_{r}, v_{\theta}, v_{\phi}$. In the phase-space the particle distribution function $f\left(r, v_{r}, v_{\theta}, v_{\phi}\right)$ evolves as dictated by the classic Boltzmann equation!

$$
\partial_{t} f+v_{r} \partial_{r} f+\dot{v}_{r} \partial_{v_{r}} f+\dot{v}_{\theta} \partial_{v_{\theta}} f+\dot{v}_{\phi} \partial_{v_{\phi}} f=\left(\partial_{t} f\right)_{\star} .
$$

Here $\left(\partial_{t} f\right)_{\star}$ is a term that describes collective 'collisions' contributing to dynamical relaxation, and will be specified in $\S 2.1$ and in the Appendix. The single-particle velocity components change according to the equations of motion

$$
\begin{aligned}
& \dot{v}_{r}=-\frac{G M(<r)}{r^{2}}+\frac{v_{\theta}^{2}+v_{\phi}^{2}}{r}, \\
& \dot{v}_{\theta}=-\frac{v_{r} v_{\theta}}{r}+\frac{v_{\phi}^{2}}{r \tan \theta}, \\
& \dot{v}_{\phi}=-\frac{v_{r} v_{\phi}}{r}-\frac{v_{\theta} v_{\phi}}{r \tan \theta},
\end{aligned}
$$

where $M(<r)$ is the DM mass within a radius $r$.

Next we derive the evolution equations for moments of the velocity distribution, up to the second order inclusive. In the following the 'mean' value $\langle X\rangle$ of a generic quantity $X$ is defined by

$$
<X>\equiv \frac{1}{\rho} \int \mathrm{d}^{3} v f X,
$$

where the spatial density is $\rho \equiv \int \mathrm{d}^{3} v f$. In the tangential directions, the mean components of the velocity $\left\langle v_{\theta, \phi}\right\rangle=0$ must vanish (as all other moments of odd order) while the corresponding dispersions $\sigma_{\theta}^{2}=\sigma_{\phi}^{2}$ must be equal (in fact, the value of any moment is unaffected by interchanging $v_{\theta}$ and $v_{\phi}$ ). In the radial direction, instead, one has to consider the mean velocity $u \equiv<v_{r}>$ and the dispersion $\sigma_{r}^{2} \equiv<\left(v_{r}-u\right)^{2}>=<v_{r}^{2}>-u^{2}$.

To obtain a closed set of moment equations we need to relate the third moment with the lowest-order ones. The standard and simplest assumption is to require zero radial skewness $\left\langle\left(v_{r}-u\right)^{3}\right\rangle=0$, which yields $\left.<v_{r}^{3}\right\rangle=u\left(u^{2}+3 \sigma_{r}^{2}\right)$; in the literature this is often referred to as 'fluid approximation' (see Teyssier et al. 1997; Chuzhoy \& Nusser 2000; Subramanian 2000; also Dehnen \& Read 2011 and references therein). Radial skewness, on the other hand, would imply an outward flow of energy through the system, or in other words a tendency for the most energetic particles to move preferentially outwards. Skewness is often taken into account

\footnotetext{
${ }^{1}$ For the sake of simplicity, in spherical symmetry and in the absence of net macroscopic rotation of the halo, one can neglect any explicit dependence of $f$ on the angular variables $\theta$ and $\phi$ (see Larson 1969, 1970; Subramanian 2000). In fact, the related terms $\left(\partial_{\theta} f\right) / r,\left(\partial_{\phi} f\right) / r \sin \theta$ to be added on the 1.h.s. of Eq. (2) would introduce in the moment equations (Eqs. 5 to be derived next) averages like $\left\langle v_{\theta, \phi}\right\rangle$ or dispersions like $\sigma_{r \theta, r \phi, \theta \phi}^{2}$ that must vanish anyway in a macroscopically spherical, nonrotating halo.
} 
in studies of star clusters' outskirts to evaluate the secular evolution of the system due to the escape of energetic stars; on the other hand, two-body encounters tend to erase skewness at the center over a timescale slightly longer than the local relaxation time, see Lynden-Bell \& Wood (1968) and Larson (1970). These analyses suggest skewness to be smaller than second-order moments by one order of magnitude or more throughout the system. In the context of DM halos accreting from a cosmological environment, it has been shown (see references at the beginning of the paragraph) that skewness is negligible in the inner region where large amount of phase-mixing has occurred, and it may become relevant only in a narrow region around the virial boundary (or better, the outermost caustics), which in the fluid approximation is considered as a sharp discontinuity and dealt with as discussed in $\S 3.1$.

Multiplying Eq. (2) by $1, v_{r},\left(v_{r}-u\right)^{2}, v_{\theta}^{2}$ and integrating over the velocity distribution yields the system of coupled partial differential equations

$$
\begin{aligned}
& \left(\partial_{t}+u \partial_{r}\right) \rho+\frac{\rho}{r^{2}} \partial_{r}\left(r^{2} u\right)=0, \\
& \left(\partial_{t}+u \partial_{r}\right) u+\frac{1}{\rho} \partial_{r}\left(\rho \sigma_{r}^{2}\right)+\frac{2}{r}\left(\sigma_{r}^{2}-\sigma_{\theta}^{2}\right)+\frac{G M(<r)}{r^{2}}=0, \\
& \left(\partial_{t}+u \partial_{r}\right) \sigma_{r}^{2}+2 \sigma_{r}^{2} \partial_{r} u=\left(\partial_{t} \sigma_{r}^{2}\right)_{\star} \\
& \left(\partial_{t}+u \partial_{r}\right) \sigma_{\theta}^{2}+\frac{2}{r} \sigma_{\theta}^{2} u=\left(\partial_{t} \sigma_{\theta}^{2}\right)_{\star} \\
& \partial_{r} M=4 \pi r^{2} \rho .
\end{aligned}
$$

These are commonly referred to as the continuity, momentum, energy, angular momentum, and mass equations; note that the second constitutes the extension of Eq. (1) to time-dependent conditions.

Remarkably, the first and the third Eqs. (5) can be combined into the form

$$
\left(\partial_{t}+u \partial_{r}\right) \log \frac{\sigma_{r}^{2}}{\rho^{2} r^{4}}=\frac{\left(\partial_{t} \sigma_{r}^{2}\right)_{\star}}{\sigma_{r}^{2}} .
$$

This describes an entropy flow for a one-dimensional fluid with effective density $\tilde{\rho} \equiv \rho r^{2}$, effective pressure $\tilde{p} \equiv$ $\tilde{\rho} \sigma_{r}^{2}$, and effective specific entropy $\tilde{s} \equiv \log \tilde{p} / \tilde{\rho}^{3}$, in terms of a microscopic adiabatic index equal to 3 .

\subsection{The 'collision' terms}

We now turn to discuss the terms $\left(\partial_{t} \sigma_{r, \theta}^{2}\right)_{\star}$ on the r.h.s. of the third and fourth Eqs. (5), that describe collective 'collisions' in the DM fluid; hereafter these will be simply referred to as collision terms. Physically, they arise because clumpiness in the infalling matter induces fluctuations of the gravitational potential that collectively affect the dynamics of DM particles. In fact, a particle effectively sees a stochastically fluctuating potential, and (consistent with the fluctuation-dissipation theorem) suffers of a dissipative drag that produces an effective 'cooling' (see Chandrasekhar 1943; Kandrup 1980; Antonuccio-Delogu \& Atrio-Barandela 1992; Del Popolo \& Gambera 1997; Ma \& Bertschinger 2004; for a review see Binney \& Tremaine 2008). On the other hand, as pointed out by Bekenstein \& Maoz (1992) and Ma \&
Boylan-Kolchin (2004), effective 'heating' of a particle by the fluctuations can also constitute a relevant process.

In the present context, we consider a halo of mass $M$ including clumps of typical mass $m$. In Appendix A we work out the form of the collision terms in the FokkerPlanck approximation, under the assumption of a quasiisotropic distribution function; the results are as follows

$$
\begin{aligned}
& \left(\partial_{t} \sigma_{r}^{2}\right)_{\star}=-\frac{64 \sqrt{3 \pi}}{15} G^{2} m \rho \log \Lambda \frac{\sigma_{r}^{2}-\sigma_{\theta}^{2}}{\sigma_{\mathrm{tot}}^{3}}, \\
& \left(\partial_{t} \sigma_{\theta}^{2}\right)_{\star}=+\frac{32 \sqrt{3 \pi}}{15} G^{2} m \rho \log \Lambda \frac{\sigma_{r}^{2}-\sigma_{\theta}^{2}}{\sigma_{\mathrm{tot}}^{3}},
\end{aligned}
$$

with $\log \Lambda \equiv \log M / m$ the standard 'Coulomb $\log$ arithm' (see Appendix A.2 for details). Note that the ratio of the average relaxation timescale $\sim$ $10^{-1} \sigma^{3} / G^{2} m \rho \log (M / m)$ from Eqs. (7) to the Hubble time $(3 / 8 \pi G \rho)^{1 / 2}$ reads $\sim$ a few $10^{-1} \mathcal{N} / \log \mathcal{N}$ in terms of the effective clump number $\mathcal{N} \equiv M / m$; this implies that relaxation can be efficient for $\mathcal{N} \lesssim 10$, i.e., when a limited number of clumps is present (a more detailed discussion of the relaxation strength is provided in Appendix A.2 and recalled in $\S 3$ ).

Note that in Eqs. (7) the (negative) cooling term dominates in the radial direction, while the (positive) heating (5) term dominates in the tangential direction. The collisions induced by clumps sharing the accretion inflow do not change the total velocity dispersion $\sigma_{\text {tot }}^{2} \equiv \sigma_{r}^{2}+2 \sigma_{\theta}^{2}$, since $\left(\partial_{t} \sigma_{\text {tot }}^{2}\right)_{\star}=0$ holds; this means that the overall random component of the energy is conserved, being just redistributed between the radial and the tangential degrees of freedom. On the other hand, collisions tend to erase any velocity anisotropy since $\left[\partial_{t}\left(\sigma_{r}^{2}-\sigma_{\theta}^{2}\right)\right]_{\star} \propto-\left(\sigma_{r}^{2}-\sigma_{\theta}^{2}\right)$ holds.

Such collective collisions provide one mechanism contributing to dynamical relaxation, close to the classic but still incomplete notion of violent relaxation (see $\S 1$ and references therein). The other mechanism is related to phase-mixing, and its implications in the present context will be discussed in $\S 3.1$.

\section{SELF-SIMILAR DESCRIPTION}

Self-similar solutions of the above Eqs. (5) obtain under two assumptions: (i) an Einstein-de-Sitter cosmological framework, which still provides an increasingly good approximation to the concordance cosmology for $z \gtrsim 0.5$ where most galactic halos form and evolve; (ii) a spherically-symmetric, scale-free shape of the initial DM mass perturbation of the form

$$
\frac{\delta M}{M} \propto M^{-\epsilon} \propto r^{-3 \epsilon},
$$

which may be considered as a piecewise approximation to a realistically bell-shaped cold DM perturbation. Here $\delta M$ represents the mass excess in a shell of initial comoving radius $r_{i} \propto M^{1 / 3}$ enclosing a mass $M$ of matter at background density. Such a shell will progressively detach from the Hubble flow, reach a maximum 'turnaround' radius $R_{\mathrm{ta}} \propto r_{i} /(\delta M / M) \propto M^{\epsilon+1 / 3}$, and collapse back to the standard virial radius $R_{200} \lesssim R_{\mathrm{ta}} / 2$.

The virialization occurs when $\delta M / M$ attains the critical threshold $1.686 D^{-1}(t)$ in terms of the linear growth 
factor $D(t) \propto t^{2 / 3}$ along the cosmic time $t$ in the Einstein-de-Sitter cosmology. So the shape parameter $\epsilon$ also governs the mass buildup after $M(t) \propto D^{1 / \epsilon}(t) \propto$ $t^{2 / 3 \epsilon}$, or equivalently sets the collapse time $t_{\text {coll }} \equiv$ $M / \dot{M}=3 \epsilon t / 2$ for the shell surrounding the mass $M$. Values $\epsilon \lesssim 2 / 3$ correspond to a stage of fast collapse relative to the Hubble expansion, while values $\epsilon \gtrsim 2 / 3$ correspond to a slow mass accretion.

The scaling $M \propto t^{2 / 3 \epsilon} \propto(1+z)^{-1 / \epsilon}$ may be conveniently compared with that of the characteristic clustering mass $M_{c} \propto(1+z)^{-6 /(n+3)}$ in a scale-free hierarchical cosmology with a power spectrum $P(k) \propto k^{n}$ (e.g., Peebles 1980). Perturbations characterized by a specific value of $\epsilon$ therefore accrete mass at the same rate as the 'typical' mass with $\epsilon=(n+3) / 6$. For example, the fast collapse of the perturbation bulk for a galactic halo is described by $\epsilon \approx 1 / 12 \div 1 / 6$ corresponding to $n \approx-2.5 \div-2$, while for the halo of a galaxy cluster values $\epsilon \approx 1 / 6 \div 1 / 3$ apply corresponding to $n \approx-2 \div-1$. On the other hand, during the development of the outskirts, the accretion rate slows down and $\epsilon$ can grow considerably larger (see discussion in $\S 5$ ).

Once the shape of the initial perturbation has been specified, there are no additional physical scales in the problem, and the time evolution of the system must approach self-similarity after a short initial transient. This implies that a single solution describes the structure and time behavior of the system, when expressed in properly scaled variables. Since the current turnaround radius is easily seen to grow as

$$
R_{\mathrm{ta}}(t) \propto t^{\xi}, \quad \text { with } \quad \xi \equiv \frac{2}{9 \epsilon}(1+3 \epsilon),
$$

it is convenient to introduce the self-similar variable $\lambda \equiv r / R_{\mathrm{ta}}(t)$, and define the adimensional radial velocity, density, mass, and velocity dispersions through the relations

$$
\begin{aligned}
& u(r, t)=\frac{R_{\mathrm{ta}}}{t} \mathcal{U}(\lambda), \\
& \rho(r, t)=\frac{1}{6 \pi G t^{2}} \mathcal{D}(\lambda), \\
& M(<r, t)=\frac{2 R_{\mathrm{ta}}^{3}}{9 G t^{2}} \mathcal{M}(\lambda), \\
& \sigma_{r, \theta}^{2}(r, t)=\frac{R_{\mathrm{ta}}^{2}}{t^{2}} \Sigma_{r, \theta}^{2}(\lambda) .
\end{aligned}
$$

Thus we can transform Eqs. (5) into the system of ordinary differential equations

$$
\begin{gathered}
(\mathcal{U}-\lambda \xi) \mathcal{D}^{\prime}+\left(\frac{2 \mathcal{U}}{\lambda}+\mathcal{U}^{\prime}-2\right) \mathcal{D}=0, \\
(\mathcal{U}-\lambda \xi) \mathcal{U}^{\prime}+(\xi-1) \mathcal{U}+\frac{1}{\mathcal{D}}\left(\mathcal{D} \Sigma_{r}^{2}\right)^{\prime}+ \\
+\frac{2}{\lambda}\left(\Sigma_{r}^{2}-\Sigma_{\theta}^{2}\right)+\frac{2}{9} \frac{\mathcal{M}}{\lambda^{2}}=0, \\
(\mathcal{U}-\lambda \xi)\left(\Sigma_{r}^{2}\right)^{\prime}+2\left(\xi-1+\mathcal{U}^{\prime}\right) \Sigma_{r}^{2}=\left(\Sigma_{r}^{2}\right)_{\star}^{\prime},
\end{gathered}
$$

$$
\begin{aligned}
& (\mathcal{U}-\lambda \xi)\left(\Sigma_{\theta}^{2}\right)^{\prime}+2\left(\xi-1+\frac{\mathcal{U}}{\lambda}\right) \Sigma_{\theta}^{2}=\left(\Sigma_{\theta}^{2}\right)_{\star}^{\prime}, \\
& \mathcal{M}^{\prime}=3 \lambda^{2} \mathcal{D},
\end{aligned}
$$

where prime denotes differentiation with respect to $\lambda$.

Moreover, with a constant value for the effective number $\mathcal{N} \equiv M / m$ of clumps in the infalling matter, the collision terms are seen to scale as $1 / t$, and hence they do not break the self-similarity. Using the variables defined in Eqs. (10) we can write them in the form

$$
\begin{aligned}
& \left(\Sigma_{r}^{2}\right)_{\star}^{\prime}=-\kappa_{\star} \frac{\mathcal{D} \mathcal{M}}{\Sigma_{\text {tot }}^{3}}\left(\Sigma_{r}^{2}-\Sigma_{\theta}^{2}\right), \\
& \left(\Sigma_{\theta}^{2}\right)_{\star}^{\prime}=+\frac{\kappa_{\star}}{2} \frac{\mathcal{D} \mathcal{M}}{\Sigma_{\text {tot }}^{3}}\left(\Sigma_{r}^{2}-\Sigma_{\theta}^{2}\right),
\end{aligned}
$$

ready to be inserted in Eqs. (11). In the above expression

$$
\kappa_{\star} \equiv \frac{64 \sqrt{3 \pi}}{405} \frac{\log \mathcal{N}}{\mathcal{N}}
$$

is the strength parameter of the collective collisions, which depends mainly on the 'clumpiness' $1 / \mathcal{N}$ of the infalling matter, see Appendix A and Fig. A1 for details.

In solving numerically the fully self-similar Eqs. (11), we will consider different values of $\kappa_{\star}$ guided by the following physical considerations. During the fast accretion $(\epsilon \lesssim 2 / 3)$ we expect that a limited number of major clumps $\mathcal{N} \lesssim 10$ rapidly merge to build up the halo bulk; after Eq. (13) this implies efficient dynamical relaxation, with strength parameter taking on values $\kappa_{\star} \approx 0.1$. On the other hand, during the late development of the outskirts we expect slow accretion $(\epsilon \gtrsim 2 / 3)$ of many small clumps with $\mathcal{N} \gtrsim 30$; these conditions imply inefficient dynamical relaxation with $\kappa_{\star} \lesssim 0.01$.

We stress that such values for the effective number of clumps in the different accretion regimes are consistent with the findings of numerical simulations (see Wang et al. 2011, their Fig. 7). The latter show that most of the mass in the inner region (which in turn is about $20 \%$ of the total) is contributed by a number of $5-10$ major mergers with mass ratios $1: 2-1: 3$; on the other hand, most of the mass in the outskirts is contributed by a number $\gtrsim 30$ of minor mergers with mass ratio $\lesssim 1: 10$, and the rest by smooth and diffuse accretion.

\subsection{Boundary conditions}

Since the original Eqs. (5) describing the DM flow are hyperbolic, discontinuities are expected to develop. However, given the collisionless nature of the DM particles (at the binary level), the discontinuities are constituted not by shocks, but rather by caustics where the bulk infall energy is partly converted into random motions. The caustics occur very close to the turning points where the radial velocity of a shell vanishes (e.g., Bertschinger 1985); there adjacent mass shells catch up with each other, and the intervening matter is compressed to a divergent density. Inward of a caustic multiple 'shell crossings' occur, the effective gravitational force experienced by a particle is (albeit slowly) time dependent, and neighboring particles' orbits go out of phase, causing the so called 'phasemixing'. Deeper in radius and/or later in time, more 
shell crossings have occurred, to originate a smoother coarse-grained particle distribution in phase-space.

In the treatments based on the Lagrangian viewpoint where the orbits of single DM particles are followed, phase-mixing primarily takes place in an outer layer including a few caustics (see Fillmore \& Goldreich 1984, Bertschinger 1985). In the fluid approximation one renders the layer of such caustics with a single 'discontinuity' across which the relevant Rankine-Hugoniot-type jump conditions apply. An extensive literature has shown the effectiveness of such an approach in closely matching the single-particle results (see Teyssier et al. 1997; Chuzhoy \& Nusser 2000; Subramanian 2000). On the other hand, in the volume inward of the discontinuity the other relaxation mechanism related to collective collisions can operate (see $\S 2.1)$.

The radius where the caustic discontinuity occurs in the self-similar description must be a constant fraction $\lambda_{c}$ of the turnaround radius, and will be computed below as an eigenvalue (see Bertschinger 1985). In detail, we proceed as follows. First of all, outside $\lambda_{c}$ the evolution is identical to the turnaround and collapse of a pressureless mass shell; a parametric form of the upstream accretion flow is given by (e.g., Peebles 1980)

$$
\begin{aligned}
& \lambda=\sin ^{2} \frac{\theta}{2}\left(\frac{\theta-\sin \theta}{\pi}\right)^{-\xi}, \\
& \mathcal{U}(\lambda)=\frac{\lambda \sin \theta(\theta-\sin \theta)}{(1-\cos \theta)^{2}}, \\
& \mathcal{D}(\lambda)=\frac{9}{2} \frac{(\theta-\sin \theta)^{2}}{(1-\cos \theta)^{3}(1+3} \\
& \mathcal{M}(\lambda)=\frac{9}{2} \lambda^{3} \frac{(\theta-\sin \theta)^{2}}{(1-\cos \theta)^{3}} .
\end{aligned}
$$$$
\mathcal{D}(\lambda)=\frac{9}{2} \frac{(\theta-\sin \theta)^{2}}{(1-\cos \theta)^{3}(1+3 \epsilon \chi)} \quad \text { with } \quad \chi=1-\frac{3}{2} \frac{\mathcal{U}(\lambda)}{\lambda},
$$

Note that at the turnaround position corresponding to $\lambda=1$ the above Eqs. (14) give $\mathcal{U}(1)=\Sigma_{r, \theta}^{2}(1)=0$, $\mathcal{M}(1)=(3 \pi / 4)^{2}$ and $\mathcal{D}(1)=\mathcal{M}(1) /(1+3 \epsilon)$.

Then, by integrating the self-similar Eqs. (11) in a region across the caustic discontinuity, we obtain the jumps

$$
\begin{aligned}
& \mathcal{U}_{+}-\xi \lambda_{c}=\frac{1}{2}\left(\mathcal{U}_{-}-\xi \lambda_{c}\right), \\
& \mathcal{D}_{+}=2 \mathcal{D}_{-} \\
& \left(\Sigma_{r}^{2}\right)_{+}=\frac{1}{16}\left(\mathcal{U}_{-}-\xi \lambda_{c}\right)^{2},
\end{aligned}
$$

here the,-+ signs refer to upstream and downstream values, respectively. Outward of the discontinuity, we have taken the radial and tangential velocity dispersion to be null, since only bulk radial motions are associated to the inflow of a shell until it collapses back to $\lambda_{c}$ and undergoes shell crossing. At the discontinuity, the flow is compressed and the bulk radial velocity is converted into radial dispersion, similarly to what occurs with the usual jump conditions at a shock but for a fluid with one degree of freedom.

On the other hand, tangential velocity dispersion is also generated by a nonspherical collapse, but a simple treatment like the present one cannot tell to what degree this occurs; in fact, no source term for the tangential dispersion is present in the corresponding moment equation, meaning that it must be assigned as a boundary condition. For example, Zukin \& Bertschinger (2010a) took steps toward estimating the tangential dispersion at the turnaround basing on tidal torque theory; their result overestimates somewhat (by a factor about 2) the outcomes of $N$-body simulations, and ultimately must be tuned in terms of the latter. This issue is beyond the scope of the present paper, and we just parameterize the tangential dispersion at the caustic discontinuity in terms of the boundary value $\beta_{+} \equiv 1-\left(\Sigma_{\theta}^{2} / \Sigma_{r}^{2}\right)_{+}$for the anisotropy parameter. We shall find that such a value does not affect the inner shapes of the mass and pseudo phase-space density profiles when efficient dynamical relaxation is at work.

Technically, the location of the caustic discontinuity $\lambda_{c}$ is determined as an eigenvalue (e.g., Bertschinger 1985), by imposing the following inner physical constraints

$$
\mathcal{M}(0)=\mathcal{U}(0)=0,
$$

i.e., the mass and velocity at the center must vanish. Note that the caustic constitutes an effective boundary for the halo, which is close if exterior to the virial radius $R_{200}$.

\section{SELF-SIMILAR SOLUTIONS}

We now turn to solving the self-similar Eqs. (11). Before handling the problem numerically, useful insights into the behavior of the solutions is found from the analytic work that follows.

\subsection{Conservation laws}

First of all, we find that integrals of motion are associated to the self-similar Eqs. (11) when collisions terms are neglected. It is convenient to introduce the auxiliary function (see Chuzhoy \& Nusser 2000)

$$
\mathcal{F}(\lambda) \equiv \exp \int^{\xi} \frac{\mathrm{d} \xi^{\prime}}{\mathcal{U}-\lambda \xi^{\prime}},
$$

so that $\mathcal{U}=\lambda \xi+\mathcal{F} / \mathcal{F}^{\prime}$ and $\mathcal{U}^{\prime}=\xi+1-\mathcal{F} \mathcal{F}^{\prime \prime} /\left(\mathcal{F}^{\prime}\right)^{2}$ obtain. Then from the continuity, mass, energy, and angular momentum equations we find

$$
\begin{aligned}
& \mathcal{D} \propto \lambda^{-2} \mathcal{F}^{1-3 \xi} \mathcal{F}^{\prime}, \\
& \mathcal{M} \propto \frac{3}{2-3 \xi} \mathcal{F}^{2-3 \xi}, \\
& \Sigma_{r}^{2} \propto \mathcal{F}^{-4 \xi}\left(\mathcal{F}^{\prime}\right)^{2}, \\
& \Sigma_{\theta}^{2} \propto \lambda^{-2} \mathcal{F}^{2(1-2 \xi)} .
\end{aligned}
$$

Rearranging these relations yields

$$
\begin{aligned}
& \mathcal{M} \propto \frac{3}{2-3 \xi} \lambda^{2} \mathcal{D}(\mathcal{U}-\lambda \xi), \\
& \frac{\Sigma_{r}^{2}}{\lambda^{4} \mathcal{D}^{2}} \propto \mathcal{M}^{\epsilon-2 / 3}
\end{aligned}
$$




$$
\Sigma_{\theta}^{2} \lambda^{2} \propto \mathcal{M}^{\epsilon+4 / 3},
$$

which constitute the 'conservation' laws of mass, effective entropy, and angular momentum. In fact, like in Eqs. (6), the quantity $\log \left[\Sigma_{r}^{2} / \lambda^{4} \mathcal{D}^{2}\right]=\log \left[\left(\mathcal{D} \lambda^{2} \Sigma_{r}^{2}\right) /\left(\mathcal{D} \lambda^{2}\right)^{3}\right]$ may be interpreted as an effective entropy for a onedimensional fluid with effective density $\mathcal{D} \lambda^{2}$, effective pressure $\left(\mathcal{D} \lambda^{2}\right) \Sigma_{r}^{2}$, and microscopic adiabatic index equal to 3 . Since the mass is a monotonically increasing function of the radius, it follows that when $\epsilon \lesssim 2 / 3$ applies such an effective entropy increases toward smaller $r$; physically, this is interpreted as an efficient relaxation of the particles during the fast collapse of the halo inner regions. On the other hand, when $\epsilon \gtrsim 2 / 3$ applies the effective entropy grows with $r$; physically, this is interpreted as a progressive stratification of the particles' orbits (or better, of the orbit apocenters) during the slow accretion that builds up the halo outskirts (see Bertschinger 1985; also Taylor \& Navarro 2001 for a similar discussion in terms of the pseudo phase-space density).

When the collision terms are efficient, only the mass conservation still applies throughout the halo.

\subsection{Asymptotic behaviors}

We now derive analytically the asymptotic behavior of the solutions near $\lambda \simeq 0$. For the sake of simplicity and with no loss of generality, we assume the following powerlaw forms of the mean radial velocity, density, and velocity dispersion: 2

$$
\begin{aligned}
& \mathcal{U} \sim \mathcal{U}_{0} \lambda, \\
& \mathcal{D} \sim \mathcal{D}_{0} \lambda^{-\gamma}, \\
& \Sigma_{r, \theta}^{2} \sim\left(\Sigma_{r, \theta}^{2}\right)_{0} \lambda^{\omega},
\end{aligned}
$$

in terms of two exponents $\gamma$ and $\omega$; correspondingly, the mass behaves as $\mathcal{M} \sim \mathcal{M}_{0} \lambda^{3-\gamma}$ with $\mathcal{M}_{0} \equiv 3 \mathcal{D}_{0} /(3-\gamma)$, and the pseudo phase-space density $\mathcal{Q} \equiv \mathcal{D} / \Sigma^{3}$ follows $\mathcal{Q} \sim \mathcal{Q}_{0} \lambda^{-\chi}$ with $\mathcal{Q}_{0} \equiv \mathcal{D}_{0} / \Sigma_{0}^{3}$ and $\chi=\gamma+3 \omega / 2$.

For such exponents, the continuity and energy equations yield the relations

$$
\begin{aligned}
& \gamma=\frac{-2+3 \mathcal{U}_{0}}{\mathcal{U}_{0}-\xi}, \\
& \omega=-2 \frac{\xi-1-\mathcal{U}_{0}}{\mathcal{U}_{0}-\xi} .
\end{aligned}
$$

In addition, when collision terms are neglected, the momentum equation writes

$\lambda \mathcal{U}_{0}\left(\mathcal{U}_{0}-1\right)+\left(\Sigma_{r}^{2}\right)_{0} \lambda^{\omega-1}\left(-\gamma+\omega+2 \beta_{0}\right)+\frac{2}{3} \frac{\mathcal{D}_{0}}{3-\gamma} \lambda^{1-\gamma} \sim 0 ;$

\footnotetext{
2 Given the constraint $\mathcal{U}(0)=0$ from Eq. (16), in principle one should write $\mathcal{U} \sim \lambda^{\nu}$ with $\nu>0$. However, it can be shown that Eqs. (11) do not admit asymptotic solutions for $\nu<1$; hence for $\nu \geq 1$ one can write $\mathcal{U} \sim \mathcal{U}_{0} \lambda$ at the first order. Moreover, for $\nu \geq 1$ the energy and angular momentum equations imply the radial and tangential dispersions to scale asymptotically in the same manner, so that one can write $\Sigma_{r, \theta}^{2} \sim \lambda^{\omega}$.
}

hereafter $\beta_{0}$ stands for the central value of the anisotropy parameter.

There are two possibilities for satisfying Eqs. (21) and (22).

- First, in Eq. (22) the exponents of the middle and last terms are negative and equal, while the coefficient of the middle term must be negative; then $\omega=-\gamma+2$ applies, and $\gamma>1+\beta_{0}$ must hold. Together with Eqs. (21), these yield $\mathcal{U}_{0} \simeq 0$ and

$$
\begin{aligned}
& \gamma=\frac{2}{\xi}=\frac{9 \epsilon}{1+3 \epsilon}, \\
& \omega=2-\frac{2}{\xi}=\frac{2-3 \epsilon}{1+3 \epsilon}, \\
& \chi=3-\frac{1}{\xi}=\frac{3}{2} \frac{2+3 \epsilon}{1+3 \epsilon}, \quad \text { for } \quad \epsilon \geq \frac{1}{3} \frac{1+\beta_{0}}{2-\beta_{0}} .
\end{aligned}
$$

This case corresponds to the late regime of slow accretion of DM particles onto a preformed halo bulk; in fact, the inner density in physical units $\rho(t) \propto \lambda^{-\gamma} t^{-2} \propto t^{-(2-\gamma \xi)}$ behaves as $\rho(t) \propto t^{0}$ so that it is independent of the time $t$, i.e., is unaffected by the outskirts growth, consistent with the two-stage formation picture.

- Second, in Eq. (22) the coefficient of the middle term is zero so that $-\gamma+\omega+2 \beta_{0}=0$ applies, while the difference $2-\gamma-\omega>0$ between the exponents of the last and middle term must be positive to imply $\gamma<1+\beta_{0}$. Together with Eqs. (21), these relations yield $\mathcal{U}_{0} \simeq 4\left[1-6 \epsilon+\beta_{0}(1+\right.$ $3 \epsilon)] /\left[9 \epsilon\left(2 \beta_{0}-5\right)\right]$ and

$$
\begin{aligned}
& \gamma=3 \frac{3 \epsilon+2+2 \beta_{0}}{3 \epsilon+7}, \\
& \omega=\frac{3(3 \epsilon+2)-2 \beta_{0}(3 \epsilon+4)}{(3 \epsilon+7)}, \\
& \chi=\frac{3}{2} \frac{(3 \epsilon+2)\left(5-2 \beta_{0}\right)}{3 \epsilon+7}, \quad \text { for } \epsilon<\frac{1}{3} \frac{1+\beta_{0}}{2-\beta_{0}} .
\end{aligned}
$$

This case corresponds to the early fast collapse of the halo bulk; in fact, the inner density in physical units behaves as $\rho(t) \propto \lambda^{-\gamma} t^{-2} \propto t^{4\left[1-6 \epsilon+\beta_{0}(3 \epsilon+1)\right] / 3 \epsilon(3 \epsilon+7)}$ so that it grows with the time $t$. We remark that the asymptotic relation $\gamma=\omega+2 \beta_{0}$ holds like in the solutions of the anisotropic Jeans equation found by Dehnen \& McLaughlin (2005) and Lapi \& Cavaliere (2009b). We also stress that the expressions of the asymptotic slopes in Eqs. (23) and (24) are continuous at $\epsilon=\left(1+\beta_{0}\right) / 3\left(2-\beta_{0}\right)$.

Note that the classic results based on assuming adiabatic invariance of the radial action may be recovered in the fluid approximation by imposing the solution to feature for $\lambda \simeq 0$ the additional regularity condition $t \partial_{r} u=\mathcal{U}^{\prime}(\lambda) \simeq 0$, or equivalently $\mathcal{U}_{0} \simeq 0$ (see Teyssier et al. 1997, Subramanian 2000). While the slope $\gamma$ given by Eq. (23) for $\epsilon \geq\left(1+\beta_{0}\right) / 3\left(2-\beta_{0}\right)$ is consistent with $\mathcal{U}_{0} \simeq 0$, that given by Eq. (24) for $\epsilon<\left(1+\beta_{0}\right) / 3\left(2-\beta_{0}\right)$ is not as $\mathcal{U}_{0}<0$ holds; thus for any $\epsilon$ in the latter range, the maximal slope $\gamma=1+\beta_{0}$ consistent with the condition $\mathcal{U}_{0} \simeq 0$ is to apply. We give three relevant examples: in a purely radial collapse with $\beta(r)=\beta_{0}=1$ the condition $\mathcal{U}_{0} \simeq 0$ would imply $\gamma=9 \epsilon /(1+3 \epsilon)$ for $\epsilon \geq 2 / 3$ and $\gamma=2$ for any $\epsilon<2 / 3$, which is the Fillmore \& Goldreich 
(1984) result; in an isotropic core with $\beta_{0}=0$ it would imply $\gamma=9 \epsilon /(1+3 \epsilon)$ for $\epsilon \geq 1 / 6$ and $\gamma=1$ for any $\epsilon<1 / 6$; for a slope $\gamma<1$ to hold at the center it would imply $\beta_{0}<0$, i.e., prevailing tangential motions (Subramanian 2000). We remark that although not satisfying the condition $\mathcal{U}_{0} \simeq 0$, our asymptotic solution given by Eqs. (24) is physical and suited to describe the fast collapse of the halo bulk; to the best of our knowledge, this was not previously known.

In Fig. 1 we plot against $\epsilon$ the inner asymptotic behaviors given by Eqs. (23) and (24) for the slopes of the density $\gamma$ and pseudo phase-space density $\chi$, for different values of $\beta_{0}$. We remark that the full solution attains its asymptotic shape quite slowly, as a result of a logarithmic convergence; e.g., the solution with $\epsilon=1 / 6$, that features a central density slope -1 , has still a slope around -1.3 at $r \approx 0.1 r_{-2}$.

When collision terms are efficient, Eqs. (23) and (24) are still valid, but the asymptotic behavior

$\Sigma_{r}^{2}-\Sigma_{\theta}^{2} \sim \exp \left[-\frac{3}{4 \gamma+3 \omega-6} \frac{\kappa_{\star}}{\xi-\mathcal{U}_{0}} \frac{\mathcal{D}_{0} \mathcal{M}_{0}}{\left(\Sigma_{\text {tot }}\right)_{0}^{3}} \lambda^{3-2 \gamma-3 \omega / 2}\right]$

applies, and enforces $\beta_{0}=1-\left(\Sigma_{r}^{2}\right)_{0} /\left(\Sigma_{\theta}^{2}\right)_{0} \simeq 0$ to hold at the center. Then from Eqs. (23) and (24) the value $\epsilon \approx$ $1 / 6$ is seen to separate the violent collapse of the inner region from the calm, inside-out growth of the outskirts. We stress that dynamical relaxation in the inner region is mainly provided by collective collisions, whilst in the outskirts it is related to phase-mixing (see also $\S 5$ ).

Note that efficient dynamical relaxation during the fast collapse stage enforces a vanishing central anisotropy $\beta_{0}=0$, while making the inner mass and pseudo phasespace density profiles only weakly dependent on both the perturbation shape parameter $\epsilon \lesssim 1 / 6$, and the outer anisotropy parameter $\beta_{+}$. In this sense, the inner halo structure turns out to be approximately universal.

\subsection{Numerical solutions}

We solve numerically the system of ordinary differential Eqs. (11) over the spatial range $10^{-4} \leq \lambda \leq 1$ with an Adams-Bashford-Moulton method of variable order, adaptive stepsize, and error control; the location of the caustic discontinuity is determined with a standard shoot-and-match technique, by requiring the solution to satisfy the inner constraints Eqs. (16), while the jump conditions Eqs. (15) are applied across the discontinuity.

As a preliminary check, in Fig. 2 we present the solution for $\epsilon=1, \beta_{+}=1$, and no collision terms $\left(\kappa_{\star}=0\right)$ corresponding to pure radial infall onto a point-mass perturbation; this is the classic case solved by Fillmore \& Goldreich (1984) and Bertschinger (1985) basing on selfsimilar particle trajectories, and equivalently by Teyssier et al. (1997) and Subramanian (2000) in the fluid approximation. We recover in detail the solutions of the latter authors.

Then we focus on the value $\epsilon=1 / 6$ that corresponds to a spectral index $n=-2$ typical of a galactic halo. Figs. 3-4-5 refer to $\beta_{+}=1, \beta_{+}=0.5$ and $\beta_{+}=0.25$, respectively, still in the absence of collisions $\left(\kappa_{\star}=0\right)$; these illustrate how the inner density profile is flattened relative to the purely radial case when non-radial motions are included. However, as already stressed by Subramanian (2000), Nusser (2001), and Zukin \& Bertschinger (2010b) such a flattening depends on the amount of angular momentum assigned at the caustic discontinuity and on how mass shells are torqued after turnaround, so that producing the approximately universal shape of the inner density profiles found in simulations requires fine tuning of a sort in the initial conditions and/or in the torque mechanism. Moreover, note that in the absence of collision terms, the anisotropy profile $\beta(r)$ rises inward from the boundary value $\beta_{+}$, a behavior at strong variance with what is seen in numerical simulations.

In Figs. 6-7-8 we retain the values $\epsilon=1 / 6$ and $\beta_{+}=$ 0.25, but include efficient collision terms; from Fig. 6 to 7 to 8 the strength parameter $\kappa_{\star}$ of collisions is increased from 0.01 to 0.05 to 0.1 . The effect of collisions is twofold: the inner slope of the density profile is now flattened to values $\gamma \lesssim 1$, while the anisotropy parameter $\beta(r)$ is lowered inward to a vanishing value $\beta_{0} \approx 0$. As collisions become more and more efficient, these effects occur on wider and wider scales. The same qualitative behavior takes place for other values of $\epsilon \lesssim 1 / 6$, as illustrated in Fig. 9 for the specific case $\epsilon=1 / 8$, and still with $\beta_{+}=0.25$ and $\kappa_{\star}=0.1$.

In Fig. 10 we plot the position of the caustic discontinuity $\lambda_{c}$ as a function of $\epsilon$, for two different values of $\beta_{+}$, with and without efficient collision terms. At fixed $\beta_{+}$ and $\kappa_{\star}$, it is seen that $\lambda_{c}$ increases with $\epsilon$; this is because as $\epsilon$ grows and the infall rate slows down, the lower infall stress allows the caustic discontinuity to be located farther out. At fixed $\epsilon$ and $\kappa_{\star}$, a lower value of $\beta_{+}$, corresponding to a higher angular momentum, yields a larger value of $\lambda_{c}$; this is because for particles with higher angular momentum it is harder to penetrate deep into the halo. Finally, at fixed $\epsilon$ and $\beta_{+}$, a higher $\kappa_{\star}$ yields a smaller $\lambda_{c}$ since collisions isotropize the velocity dispersions, and so the particles lose part of their initial angular momentum and can penetrate deeper into the halo.

We stress that all the above self-similar solutions feature a wide radial range from the center to about a few $r_{-2}$ where the bulk velocity $u$ is approximately null. This implies that in the second of Eqs. (5) the term $\left(\partial_{t}+u \partial_{r}\right) u \simeq 0$ closely vanishes, and the equation itself reduces to a Jeans form like Eq. (1). As such it describes a nearly static equilibrium, endowed with runs of $Q(r)$ and $\beta(r)$ as provided by the full system of equations.

\subsection{Comparison with $N$-body simulations}

In Figs. 11-14 we compare our self-similar solutions to the outcomes of state-of-the-art numerical simulations. In all these plots, for the solution inward of $r_{-2}$ our fiducial values are $\epsilon=1 / 8$ (as representative for the range $\epsilon \lesssim 1 / 6)$ and $\kappa_{\star}=0.1$; in fact, these apply to the fast inner collapse of galactic halos with effective spectral index $n=6 \epsilon-3 \approx-2.25$, when rapid merging of an effective number of clumps $\mathcal{N} \lesssim 10$ implies efficient dynamical relaxation (see $\S 3$ and Fig. A1). For the solution outward of $r_{-2}$, our fiducial values are $\epsilon=1 / 2$ (as representative for the range $\epsilon \gtrsim 1 / 6$ ) and $\kappa_{\star}=0.01$. This is because we expect the outskirts growth to be dominated by smooth accretion from the tapering perturbation wings with effective spectral index $n=6 \epsilon-3 \approx 0$; now the accretion involves many small clumps with $\mathcal{N} \gtrsim 30$, implying dynamical relaxation to become inefficient. We stress that similar behaviors obtain in the two radial ranges for reasonable variations of $\epsilon$ and $\kappa_{\star}$ around our reference 
values. The overall picture to be compared with real or simulated data may be obtained on matching these two solutions around $r_{-2}$.

As to the other parameter $\beta_{+}$, this is less amenable to physical pinpointing. We expect $\beta_{+}<1$, i.e., a deviation from purely-radial collapse, due to asymmetries both in velocity and configuration space. On the other hand, from the experimentations reported in $\S 4.3$ we know that in the presence of $\kappa_{\star} \gtrsim 0.01$, specific boundary values of $\beta_{+}$do not materially affect the solution, including the run of $\beta(r)$ vanishing toward the center. Finally, note that around the outer caustic discontinuity $\beta=1-\Sigma_{\theta}^{2} / \Sigma_{r}^{2}$ is ill defined anyway, since both the radial and the tangential dispersions are small upstream (see also end of this section). Within the above constraints, we adopt the value $\beta_{+}=0.25$ both for the inner and outer solutions, because around $r_{-2}$ this yields similar values of $\beta$, and provides a close match of the two (already comparable) density slopes.

In Fig. 11 we compare the inner self-similar profiles of density (top panels) and density slope (bottom panels) with standard fitting formulae to the outcomes of numerical simulations. Specifically, we illustrate the NFW profile (dot-dashed line) and the Einasto profile with shape parameter $\eta=0.17$ (dashed line); in the inner radial range $r \gtrsim 0.04 r_{-2}$ that is currently accessible to numerical simulations, the former constitutes a popular description of the virtual data, while the latter has been recently found to constitute a better functional representation (see $\S 1$ ). It is seen that the inner self-similar solution follows closely the Einasto profile for $r \gtrsim 0.01 r_{-2}$, but for smaller radii deviates to attain a central asymptotic slope -0.86 (see $\S 4.2$ and Fig. 1); in other words, a steepening of the density profile is predicted for radii $r \lesssim 0.01 r_{-2}$ relative to the flat Einasto shape. In Fig. 11 we show that a substantially better representation of the self-similar solution over the whole range $r<r_{-2}$ is provided by the Sérsic-Einasto formula (as recalled in $\S 1$; cf. also with Graham et al. 2006) with shape parameters $\tau=0.9$ and $\eta=0.35$. It will be interesting to test such a behavior with numerical experiments of higher resolutions than presently achieved.

In Fig. 12 we compare the density profile $\rho(r)$ of the self-similar solutions (thick black solid line) to the outcomes for six different equilibrium halos extracted from the Aquarius $N$-body simulation (Navarro et al. 2010; thin colored lines); an Einasto profile with shape parameter $\eta=0.17$ is also shown for reference (dashed line). The agreement of the self-similar solutions with the simulation results is remarkably good for $r \leq$ a few $r_{-2}$. A discrepancy occurs in the outer regions on approaching the caustic discontinuity; this is expected since our fluid approximation breaks down there, while the simulated halos themselves may be out of equilibrium.

In Fig. 13 we compare the self-similar profiles (thick black solid line) of phase-space density $Q(r)$ to the outcomes from the same six Aquarius halos plotted in the previous Figure (Ludlow et al. 2010; thin colored lines); the powerlaw $Q(r) \propto r^{-1.875}$ is also shown for reference (dashed line). The self-similar profiles agree with the simulation results as to a close powerlaw shape for $0.04 r_{-2} \lesssim r \lesssim$ a few $r_{-2}$. For radii $r \lesssim 0.01 r_{-2}$ that are not presently probed by simulations, the self-similar so- lutions predict a steepening of $Q(r)$ to attain an asymptotic slope around 2.4 (as expected on the basis of the asymptotic analysis of $\S 4.2$, see Fig. 1; cf. also with Graham et al. 2006); it will be important to test such a behavior with future simulations of higher resolution than currently achieved.

The fact that the powerlaw behavior found in simulations is close to $Q(r) \propto r^{-1.875}$ over an extended range has often been considered surprising and puzzling; in fact, the value -1.875 marks the classic self-similar solution by Bertschinger (1985) for the purely radial collapse of a point-mass perturbation. On the other hand, the effective spectral index $n=3$ corresponding to the Bertschinger's solution constitutes a poor approximation of the typical overdensity initiating galactic DM halos; in addition, the resulting inner density profile is quite steeper than found in galaxy simulations (as recalled in $\S 1)$. Here we see that a powerlaw behavior close to $Q(r) \propto r^{-1.875}$ in the halo middle is actually featured by our self-similar solutions with more realistic values $n \approx-2.25$, when non-radial motions and collision effects are included; we find that such a powerlaw behavior extends over a considerable radial range around $r_{-2}$. Inward of this, $Q(r)$ logarithmically steepens while the density asymptotes to slopes $\lesssim 0.9$; at the other end, beyond $r_{-2}$ toward the caustic discontinuity $Q(r)$ features an upturn, for which preliminary evidence has also emerged in recent numerical studies (see Ludlow et al. 2010).

In Fig. 14 we compare the self-similar anisotropy profile (thick black solid line) with the outcomes from the same six Aquarius halos plotted in the previous Figures (Ludlow et al. 2010; thin colored lines); the outcome obtained from the Einasto profile with $\eta=0.17$ and the Hansen \& Moore (2006) $\beta-\gamma$ relation is also illustrated for reference (dashed line). Once again, the self-similar solutions agree with the simulation results (cf. also with the observations by Biviano \& Poggianti 2009). Note that close to the (outer) caustic discontinuity wide oscillations in the $\beta=1-\sigma_{\theta}^{2} / \sigma_{r}^{2}$ parameter are found in simulations, as expected considering that both the upstream radial and tangential velocity dispersions are small.

\section{DISCUSSION AND CONCLUSIONS}

Sharing the widespread drive toward understanding the key processes that rule the dark matter (DM) component of cosmic structures from galaxies to their systems, we have investigated the detailed structure and evolution of cold DM halos. To complement the intensive and extensive numerical simulations, here we have developed advanced models of spherical collapse and accretion in an expanding Universe, based on solving timedependent equations for the moments of the phase-space distribution function in the fluid approximation. Our approach includes non-radial random motions, and most importantly, an advanced treatment of dynamical relaxation effects; thus we provide an effective description of the halo dynamical evolution toward equilibrium and of the resulting density and velocity structure. Deferring to $\S 5.5$ an overall picture of halo structure and development, we discuss first our answers to the hot issues on DM dynamics recalled in $\S 1$.

\subsection{Dynamical relaxation}


Dynamical relaxation involves two mechanisms: phasemixing, due to spreading of neighboring particle orbits in phase-space; and violent relaxation, due to irregular fluctuations of the gravitational potential. Phasemixing can be traced back to the process of shell crossing that underlies the classic self-similar treatments (see Bertschinger 1985; also Henriksen \& Widrow 1999). In our fluid approach this is expressed by the left-hand sides of Eqs. (11), with action primarily confined to a skin region close to the outer caustic; this circumstance enables one to describe its effect in terms of boundary jump conditions of Rankine-Hugoniot type. Violent relaxation, instead, is related to collective collisions, expressed by the terms on the right-hand side of the third and fourth Eqs. (11), and active throughout the volume of the halo.

The collision terms have been evaluated quantitatively with the help of a Fokker-Planck approximation in velocity space, under conditions of a closely isotropic distribution function (see Appendix A2). To derive the circularly-smoothed density run $\rho(r)$, the second Eq. (5) shows that it is important to understand the behavior of the velocity dispersions $\sigma_{r, \theta}^{2}$; to that effect, we stress that the structure of our Fokker-Planck coefficients ensures erasure of velocity anisotropy after $\left[\partial_{t}\left(\sigma_{r}^{2}-\sigma_{\theta}^{2}\right)\right]_{\star} \propto$ $-\left(\sigma_{r}^{2}-\sigma_{\theta}^{2}\right)$, while still conserving the overall random energy after $\left(\partial_{t} \sigma_{\theta}^{2}\right)_{*}=-\left(\partial_{t} \sigma_{r}^{2}\right)_{*} / 2$, see end of $\S 2.1$.

Anisotropy is initiated at the caustic discontinuity as described by the parameter $\beta_{+}$, which specifies the degree of non-radial DM motions endowed, or acquired during the initial infall. The efficiency of their erasure is modulated by the strength of the collision terms as expressed by the parameter $\kappa_{\star} \lesssim 0.1$; this reflects the amount of gravitational fluctuations induced by clumpiness in the infalling matter, well beyond the tiny levels associated to microscopic graininess. Note that dynamical relaxation acts like an effective 'torque' mechanism after turnaround, in the same vein entertained by Zukin \& Bertschinger (2010a, 2010b); in fact, these authors find results qualitatively similar to ours in their parametric torque models with decreasing angular momentum and vanishing central anisotropy.

On these grounds, we have found self-similar solutions for the spherically-averaged mass density $\rho(r)$, pseudo phase-space density $Q(r) \equiv \rho / \sigma^{3}$ and anisotropy parameter $\beta(r) \equiv 1-\sigma_{\theta}^{2} / \sigma_{r}^{2}$. Overall profiles are obtained on matching (as discussed in detail in $\S 4.4$ ) the self-similar solutions corresponding to two different portions of the initial DM perturbation, representative of the fast inner collapse and of the slow outskirt buildup. We have compared these overall profiles with the outcomes of state-ofthe-art $N$-body simulations throughout the radial range currently probed by the latter, finding a pleasing agreement.

\subsection{Halo inner regions}

Specifically, in the region inward of a few times $r_{-2}$ (the radius where the density slope is -2 ) we have found that efficient dynamical relaxation is the key process to produce the following features: a closely universal density profile in agreement with the $N$-body outcomes, well represented in terms of the Einasto formula with shape parameter $\eta \approx 0.17$; a pseudo phase-space density profile with the powerlaw behavior close to $Q(r) \propto r^{-1.9}$; and an anisotropy profile decreasing inward from values around 0.25 at $r_{-2}$ to values $\beta \approx 0$ at the center.

In the very central region $r \lesssim 0.04 r_{-2}$, currently not accessible to $N$-body simulations, we predict a steepening of the mass density profile relative to the flat Einasto shape, that would imply a vanishing central slope; the expected asymptotic behavior, though with a logarithmically slow convergence, reads $\rho(r) \propto r^{-0.86}$, to the effect that the self-similar density profile is best described by a Sérsic-Einasto formula with shape parameters $\tau=0.9$ and $\eta=0.35$. In parallel, the pseudo phase-space density steepens to approach a central behavior $Q(r) \propto r^{-2.4}$. Testing these predictions will require $N$-body simulations of higher resolution than presently achieved.

\subsection{Inner baryonic effects}

Clearly, both our self-similar solutions and $N$-body simulations refer to pure DM structures. The reader must be aware that in the central regions of real galaxies small-scale dynamics or energetics related to the astrophysics of baryons may alter the baseline DM profiles discussed above. These baryonic processes may reconcile the outcomes of simulations and self-similar models with the observations of galactic dynamics in spiral galaxies (especially dwarfs; see Salucci et al. 2007, 2011) and of gravitational lensing in galaxies (see Bradač et al. 2009) and galaxy systems (see Zitrin et al. 2011; Newman et al. 2011), which indicate approximately flat density profiles already inward of $0.1 r_{-2}$.

For example, flattening of the inner density profile may be caused by transfer of energy and/or angular momentum from (baryonic and DM) clumps to the DM field during the galaxy formation process (see El-Zant et al. 2001; Ma \& Boylan-Kolchin 2004; Tonini et al. 2006). In detail, upon transfer of tangential random motions from the baryons to an initially isotropic DM structure, the original density profile $\rho \propto r^{-\gamma}$ in the inner region is expected to be modified into $\rho \propto r^{-2\left[\gamma+2(2-\gamma) \beta_{0}\right] /\left[2+(2-\gamma) \beta_{0}\right]}$; thus the final profile is flattened for $\beta_{0}<0$, down to the point of developing a core for $\beta_{0} \lesssim-\tau / 2(2-\gamma) \approx-0.3$. However, a reliable assessment of the amount of angular momentum transferred from the baryons to the DM is still wanting, and would require aimed numerical simulations.

Less agreed processes may affect galactic scales $r \lesssim 10^{2}$ pc; for example, at the formation of a spheroid, central starbursts and accretion onto a supermassive black hole may easily discharge enough energy $\left(\sim 10^{62} \mathrm{erg}\right.$ for a black hole mass $\left.M_{\bullet} \sim 10^{9} M_{\odot}\right)$ with sufficient coupling $(\gtrsim 5 \%)$ to blow most of the gaseous baryonic mass within $r_{-2}$ out of the inner gravitational well. This will cause an expansion of the DM and of the stellar distributions (see Fan et al. 2008, 2010; Ragone-Figueroa \& Granato 2011), so as to flatten the central DM slope. In addition, binary black hole dynamics following a substantial merger may eject on longer timescales formed stars from radii $r \approx 10\left(M_{\bullet} / 10^{8} M_{\odot}\right)^{1 /(3-\tau)}$ pc containing an overall mass of a few times the black hole's, and so may cause the light deficit observed in some galaxy cores (see Graham 2004; Lauer et al. 2007; Kormendy et al. 2009).

On the other hand, some steepening of the inner density profile may be induced by any 'adiabatic' contraction of the diffuse star-forming baryons into a disc-like structure, as proposed by Blumenthal et al. (1986) and 
Mo, Mao \& White (1998). On the basis of the standard treatments, it is easily shown that in the inner region an initial powerlaw $\rho \propto r^{-\gamma}$ is turned into $\rho \propto r^{-3 /(4-\gamma)}$. However, recent numerical experiments (see Abadi et al. 2010) suggest that the classic treatment of adiabatic contraction is likely to be extreme; actually, in the inner region the contraction may be inefficient and the density slope hardly modified.

All these issues are beyond the scope of the present paper.

\subsection{Halo outskirts}

At the other end, in the region outward of a few times $r_{-2}$ we find that self-similar solutions with larger $\epsilon \gtrsim 1 / 2$ and less efficient dynamical relaxation apply, consistent with the outcomes of numerical simulations. This is expected, since the outskirts are built up at later times by smoother (i.e., less clumpy) accretion fed on the tapering wings of a DM perturbation. We note that, as highlighted by the scatter in the $N$-body results, the outskirts structure is subject to a large variance, related to the detailed growth histories and to environmental conditions wherefrom the infall takes place.

Relatedly, for redshifts $z \lesssim 0.5$ the accelerating cosmology slows down the time dependence $D(t) \propto t^{d}$ of the growth factor from $d=2 / 3$ to $d \approx 1 / 2$. A strictly self-similar solution cannot be obtained in such conditions, but the overall trend can be captured from noting that the accretion rate scales as $\dot{M} / M=d / \epsilon t$; thus a lower $d$ is equivalent to higher effective values of $\epsilon$, which result in steeper density profiles into the outskirts. Moreover, in a flat accelerating Universe, shells will be able to turnaround and collapse only if their initial position $r_{i}$ is inside a critical radius $r_{\Lambda}$ defined by $\delta M / M\left(<r_{\Lambda}\right) \equiv 3 / 2\left(2 \Omega_{\Lambda}\right)^{1 / 3}$ in terms of the dark energy density parameter $\Omega_{\Lambda} \approx 0.7$; Subramanian et al. (2000) have shown that the resulting outer density profile is considerably steep, featuring a cutoff toward $r_{\Lambda}$.

Our self-similar solutions concur with numerical simulations in providing a firm basis and in clarifying the limitations for the simple dynamical models based on the Jeans equation and on a consistent powerlaw shape for $Q(r) \propto r^{-1.9}$, see $\S 1$ for details. We find that such models are reliable in the significant range $0.01 r_{-2} \lesssim r \lesssim \mathrm{a}$ few $r_{-2}$; outside of this, in the outskirts $Q(r)$ flattens appreciably and is liable to cosmogonical variance, while in the inner regions it steepens, though logarithmically. It may be interesting to introduce in the dynamical models this articulated behavior of $Q(r)$, to investigate how the Jeans profiles are affected (taking up from the work by Lapi \& Cavaliere 2009b).

\subsection{An overall picture of halo structure and development}

Finally, we stress the link between the halo structure and its two-stage growth history from the vantage point of our self-similar solutions, to provide the following overall picture (illustrated in the schematics of Fig. 15).

The halo formation history starts up with the fast collapse of a few merging clumps, that simulations indicate to be the main contributors to the inner halo mass (see Fakhouri et al. 2010; Genel et al. 2010; Wang et al. 2011); such a strong clumpiness enforces efficient dynamical relaxation, the key process to produce an approximately universal shape of the inner density profile, and an isotropic inner halo structure.

As discussed in $\S 3$, the typical halo mass and radius scale as $M \propto t^{2 / 3 \epsilon}$ and $R \propto t^{2 / 3+2 / 9 \epsilon}$ in terms of the perturbation shape parameter $\epsilon$, or of the corresponding effective index of the perturbation spectrum $n \approx 6 \epsilon-3$. Thus the fast collapse of the inner region affected by violent relaxation is seen to correspond to $\epsilon \lesssim 1 / 6$ or $n \lesssim-2$, since for these values the depth of the gravitational well expressed by $v_{c} \sim(G M / R)^{1 / 2} \propto t^{2 / 9 \epsilon-1 / 3}$ grows faster than the cosmic time. These values are also consistent with the asymptotic analysis of $\S 4.2$. We also note that for $n \lesssim 1$ or $\epsilon \lesssim 2 / 3$ the typical specific energy $v_{c}^{2} \propto M^{2 / 3-\epsilon}$ increases with mass, so that the inner region provides an environment conducive to eventual melting of the accreted massive clumps. This validates spherically-averaged densities, and is consistent with the findings from numerical simulations that the residual mass fraction still locked into clumps is limited in the inner regions (see Springel et al. 2008, their Figs. 11-12). Note that the melting of clumps due to tidal disruption and dynamical friction may be even more efficient in a warm DM framework (e.g., Colín et al. 2000).

Subsequently, a stage of calmer and smoother accretion ensues; this is slower relative to the cosmic expansion when $n \gtrsim 1$ or $\epsilon \gtrsim 2 / 3$, since the growth rate scales as $M / t \propto t^{2 / 3 \epsilon-1}$. During this stage, simulations indicate that most of the mass is gathered about equally from minor mergers of many small clumps and from truly diffuse accretion (see above references). Then the outskirts build up from the inside-out, while the preformed inner region is nearly unaffected; this is because the smoothness of the accretion makes dynamical relaxation inefficient and only mild phase-mixing occurs at the outer caustic (see $\S 3$ and 4.2). Meanwhile, cosmological variance and non-radial motions related to the halos' specific growth history or environmental conditions become relevant in shaping the halo outskirts, in ways expected to be far from universal.

We envisage such a two-stage framework of halo growth to be of much relevance for the astrophysics of galaxy clusters, especially in their outskirts as discussed by Lapi et al. (2010), and for galaxy/star formation theories; e.g., in the latter context it is at the hearth of the twophase galaxy formation picture first proposed by Cook et al. (2009). This envisages that the two modes of halo growth drive two distinct modes for the evolution of baryonic matter, with the development of the spheroidal component of galaxies taking place mainly during the early fast collapse, and that of the disc component during the late slow accretion.

Work supported in part by ASI, INAF and MIUR. We thank our referee for helpful comments and suggestions. We acknowledge useful discussions with L. Danese, G. De Zotti, G.L. Granato, and P. Salucci. AL thanks SISSA for warm hospitality. 


\section{REFERENCES}

Abadi, G., et al. 2010, MNRAS, 407, 435

Antonuccio-Delogu, V., \& Atrio-Barandela, F. 1992, ApJ, 392, 403

Arad, I., \& Lynden-Bell, D. 2005, MNRAS, 361, 385

Ascasibar, Y., \& Binney, J. 2005, MNRAS, 356, 872

Ascasibar, Y., \& Gottlöber, S. 2008, MNRAS, 386, 2022

Austin C.G., et al. 2005, ApJ, 634, 756

Bertschinger, E. 1985, ApJS, 58, 39

Bekenstein, J.D., \& Maoz, E. 1992, ApJ, 390, 79

Binney, J. 1978, MNRAS, 183, 779

Binney, J., \& Tremaine, S. 2008, Galactic Dynamics, $2^{\text {nd }}$ edition (Princeton: Princeton Univ. Press)

Biviano, A., \& Poggianti, B.M. 2009, A\&A, 501, 419

Blumenthal, G.R., et al. 1986, ApJ, 301, 27

Boylan-Kolchin, M., Ma, C.-P., \& Quataert, E. 2008, MNRAS, 383, 93

Bradač, M., et al. 2009, ApJ, 706, 1201

Bullock, J.S., et al. 2001, MNRAS, 321, 559

Chandrasekhar, S. 1943, ApJ, 97, 255

Chuzhoy, L. \& Nusser, A. 2000, MNRAS, 319, 797

Ciotti, L. 2010, in 'Plasmas in the Laboratory and the Universe: Interactions, Patterns, and Turbulence', eds. G. Bertin, F. De Luca, G. Lodato, R. Pozzoli, and M. Romé, AIP Conf. Proc. 1242, 117

Colín, P., Avila-Reese, V., \& Valenzuela, O. 2000, ApJ, 542, 622

Cook, M., Lapi, A., \& Granato, G.L. 2009, MNRAS, 397, 534

Del Popolo, a., \& Gambera, M. 1997, A\&A, 321, 691

Dehnen, W., \& Read, J.I. 2011, Europ. Phys. J. Plus, 126, 55

Dehnen, W., \& McLaughlin, D.E. 2005, MNRAS, 363, 1057

Diemand, J., Kuhlen, M., \& Madau, P. 2007, ApJ 667, 859

Einasto, J. 1965, Trudy Inst. Astroz. Alma-Ata, 5, 87

El-Zant, A., et al. 2001, ApJ, 560, 636

Fakhouri, O., Ma, C.-P., \& Boylan-Kolchin, M. 2010, MNRAS, 406, 2267

Fan, L., et al. 2010, ApJ, 718, 1460

Fan, L., et al. 2008, ApJ, 689, L101

Fillmore, J.A. \& Goldreich, P. 1984, ApJ, 281, 1

Genel, S., Bouché, N., Naab, T., Sternberg, A., \& Genzel, R. 2010, ApJ, 719, 229

Graham, A.W., et al. 2006, AJ, 132, 2701

Graham, A.W. 2004, ApJ, 613, L33

Gunn, J.E., \& Gott, J.R. 1972, ApJ, 176, 1

Hansen, S.H., \& Moore, B. 2006, New Astronomy, 11, 333

Henriksen, R.N., \& Widrow, L.M. 1999, MNRAS 302, 321

Hoffman, Y., Romano-Díaz, E., Shlosman, I. , \& Heller, C. 2007, ApJ, 671, 1108

Huss, A., Jain, B., \& Steinmetz, M. 1999, ApJ, 517, 64

Kandrup, H.E. 1980, Phys. Rep., 63, 1

Kormendy, J., et al. 2009, ApJS, 182, 216

Lapi, A., \& Cavaliere, A., 2011, Adv. in Astron., vol. 2011, id. 903429, 7

Lapi, A., Fusco-Femiano, R., \& Cavaliere, A. 2010, A\&A, 516, 34

Lapi, A., \& Cavaliere, A., 2009a, ApJ, 692, 174

Lapi, A., \& Cavaliere, A., 2009b, ApJ, 695, L125

Larson, R.B. 1970, MNRAS, 147, 323

Larson, R.B. 1969, MNRAS, 145, 405

Lauer, T.R., et al. 2007, ApJ, 664, 226

Lu, Y., Mo, H. J., Katz, N., \& Weinberg, M. D. 2006, MNRAS, 368,1931

Ludlow, A.D., et al. 2010, MNRAS, 406, 137

Ludlow, A.D., et al. 2011, MNRAS, 415, 3895

Lithwick, Y., \& Dalal, N. 2011, ApJ, 734, 100
Lynden-Bell, D. 1967, MNRAS, 136, 101

Lynden-Bell, D., \& Wood, R. 1968, MNRAS, 138, 495

Ma, C.-P., \& Bertschinger, E. 2004, ApJ, 612, 28

Ma, C.-P., \& Boylan-Kolchin, M. 2004, Phys. Rev. Lett., 93, 1301

Merritt, D., Graham, A.W., Moore, B., Diemand, J., \& Terzić, B. 2006, AJ, 132, 2685

Mo, H.J., Mao, S., \& White, S.D.M. 1998, MNRAS, 295, 319

Nakamura, T.K. 2000, ApJ, 531, 739

Navarro J.F., et al. 2010, MNRAS, 402, 21

Navarro, J.F. et al. 2004, MNRAS, 349, 1039

Navarro, J.F., Frenk, C.S. \& White, S.D.M. 1997, ApJ, 490, 493

Newman, A.B., Treu, T., Ellis, R.S., \& Sand, D.J. 2011, ApJ, 728, L39

Nusser 2001, A. 2001, MNRAS, 325, 1397

Peebles, P.J.E. 1980, The Large-Scale Structure of the Universe (Princeton: Princeton Univ. Press)

Prada, F., et al. 2011, MNRAS, submitted (preprint arXiv:1104.5130)

Prugniel, PH. \& Simien, F. 1997, A\&A, 321, 111

Ragone-Figueroa, C., \& Granato, G.L. 2011, MNRAS, 414, 3690

Ryden, B.S. 1993, ApJ, 418, 4

Rosenbluth, M.N., MacDonald, W.M., \& Judd, D.L. 1956, Phys. Rev. 107, 1

Salucci, P., et al. 2007, MNRAS, 378, 41

Salucci, P., Frigerio-Martins, C., Lapi, A., 2011, see http://www.sissa.it/ap/dmg/index.html (preprint arXiv1102.1184)

Schmidt, K.B., Hansen, S. H., \& Macció, A.V. 2008, ApJ, 689, L33 Sedov, L.I. 1959, Similarity and Dimensional Methods in Mechanics (New York: Academic)

Sharma, S., \& Steinmetz, M. 2006, MNRAS, 373, 1293

Sérsic, J.-L. 1963, Boletin de la Asociacion Argentina de Astronomia, 6, 41

Sikivie, P., Tkachev, I.I. \& Wang, Y. 1997, Phys. Rev. D, 56, 1863 Springel, V., et al. 2008, MNRAS, 391, 1685

Springel, V., et al. 2005, MNRAS, 364, 1105

Stadel, J., et al. 2009, MNRAS, 398, L21

Subramanian, K., Cen , R., \& Ostriker, J.P. 2000, ApJ, 538, 528

Subramanian, K. 2000, ApJ, 538, 517

Taylor, J.E., \& Navarro J.F., 2001, ApJ, 563, 483

Teyssier, R., Chièze, J.-P., \& Alimi, J.-M. 1997, ApJ 480, 36

Tonini, C., Lapi, A., \& Salucci, P. 2006, ApJ, 649, 591

Trenti, M., Bertin, G., \& van Albada, T.S. 2005, A\&A, 433, 57

van Albada, T.S. 1982, MNRAS, 201, 939

Vass, I. M., Valluri, M., Kravtsov, A.V., \& Kazantzidis, S. 2009, MNRAS, 395, 1225

Vogelsberger, M., Mohayaee, R., \& White, S.D.M. 2011, MNRAS, 414,3044

Wang, J., et al. 2011, MNRAS, 413, 1373

Wang, J., \& White S.D.M., 2009, MNRAS, 396, 709

White, S.D.M. \& Zaritsky, D. 1992, ApJ, 394, 1

Zel'dovich, Ya.B., \& Raizer, Y.P. 1967, Physics of Shock Waves and High-Temperature Hydrodynamic Phenomena (New York: Academic)

Zhao, D. H., Mo, H. J., Jing, Y. P., \& Börner, G. 2003, MNRAS, 339,12

Zitrin, A., et al. 2011, MNRAS, 410, 1939

Zukin, P., \& Bertschinger, E. 2010a, Phys. Rev. D, 82, 104044

Zukin, P., \& Bertschinger, E. 2010b, Phys. Rev. D, 82, 104045

\section{APPENDIX}

THE COLLISION TERMS

In this Appendix we evaluate the collision terms to be inserted in Eqs. (5) and (11). We take up and adapt to the present problem the approach developed by Larson $(1969,1970)$ in the context of stellar clusters.

\section{The velocity distribution function}

First of all, we specify the form of the velocity distribution function. We introduce spherical polar coordinate in velocity space, and write the velocity components as

$$
v_{r}-u=\nu \mu
$$




$$
v_{\theta}^{2}+v_{\phi}^{2}=\nu^{2}\left(1-\mu^{2}\right)
$$

in terms of the random velocity vector $\nu$ and the cosine $\mu$ of the angle between this vector and the radial direction.

We assume that the normalized distribution $f(\nu, \mu)$ of random velocities is approximately isotropic, and expand it in Legendre polynomials up to the second order

$$
f(\nu, \mu) \simeq f_{0}(\nu) P_{0}(\mu)+f_{1}(\nu) P_{1}(\mu)+f_{2}(\nu) P_{2}(\mu)
$$

the coefficients $f_{1}$ and $f_{2}$ are assumed to be small corrections to the leading term $f_{0}(\nu)$ provided for definiteness by a Maxwellian-like distribution.

The normalization conditions for $f$ require that

$$
\int \mathrm{d}^{3} \nu f=1 \quad, \quad \int \mathrm{d}^{3} \nu \nu f=0
$$

hold, with $\int \mathrm{d}^{3} \nu=2 \pi \int_{0}^{\infty} \mathrm{d} \nu \nu^{2} \int_{-1}^{1} \mathrm{~d} \mu$ being the volume element. These imply that the terms in the expansion Eqs. (A2) must depend only on the combinations $\nu^{2}$ or $\nu \mu$; thus we base on the expressions

$$
\begin{array}{ll}
f_{0}(\nu)=\frac{1}{\left(2 \pi \sigma^{2}\right)^{3 / 2}} \exp \left(-\frac{\nu^{2}}{2 \sigma^{2}}\right) & P_{0}(\mu)=1 \\
f_{1}(\nu)=c_{1} \nu f_{0}(\nu) & P_{1}(\mu)=\mu \\
f_{2}(\nu)=c_{2} \nu^{2} f_{0}(\nu) & P_{2}(\mu)=\frac{3 \mu^{2}-1}{2},
\end{array}
$$

where $c_{1}$ and $c_{2}$ are constants to be determined.

As discussed in $\S 2$, to close the moment equations we are interested in a distribution with zero skewness, which gives $c_{1}=0$. On the other hand, $c_{2}$ can be determined by considering the second-order moments of the distribution, i.e.,

$$
\begin{aligned}
& \sigma_{r}^{2}=\int \mathrm{d}^{3} \nu \nu^{2} \mu^{2} f=\sigma^{2}+2 c_{2} \sigma^{4}, \\
& \sigma_{\theta}^{2}=\int \mathrm{d}^{3} \nu \nu^{2}\left(1-\mu^{2}\right) f=\sigma^{2}-c_{2} \sigma^{4} ;
\end{aligned}
$$

since $\sigma_{\text {tot }}^{2} \equiv \sigma_{r}^{2}+2 \sigma_{\theta}^{2}=3 \sigma^{2}$ and $\sigma_{r}^{2}-\sigma_{\theta}^{2}=3 c_{2} \sigma^{4}$ hold, we find

$$
c_{2}=3 \frac{\sigma_{r}^{2}-\sigma_{\theta}^{2}}{\sigma_{\text {tot }}^{4}} \text {. }
$$

\section{Fokker-Planck approximation}

Now we turn to the form of the collision terms. In the Fokker-Planck approximation, these are written as

$$
\Gamma_{\star}^{-1}\left(\partial_{t} f\right)_{\star}=-\sum_{i} \partial_{v_{i}}\left(f \partial_{v^{i}} h\right)+\frac{1}{2} \sum_{i, j} \partial_{v_{i} v_{j}}^{2}\left(f \partial_{v^{i} v^{j}} g\right)
$$

in terms of

$$
\begin{aligned}
& h=2 \int \mathrm{d}^{3} \nu^{\prime} \frac{f\left(\nu^{\prime}\right)}{\left|\nu-\nu^{\prime}\right|}, \\
& g=2 \int \mathrm{d}^{3} \nu^{\prime} f\left(\nu^{\prime}\right)\left|\nu-\nu^{\prime}\right| .
\end{aligned}
$$

The quantity $\Gamma_{\star} \equiv 4 \pi G^{2} m \rho \log \Lambda$ represents the strength parameter of the collective collisions, and depends on the clump mass $m$ as $m \log M / m$; here the standard 'Coulomb' $\log$ arithm $\log \Lambda$ has been conveniently written in terms of the ratio $M / m$ between the halo and the clump masses (see Boylan-Kolchin et al. 2008). In fact, numerical simulations indicate that the clumps (technically, 'subhalos') in a halo are distributed close to $m^{-2}$ (see Springel et al. 2008); averaging $\Gamma_{\star}$ over such a distribution turns out to be closely equivalent to redefine $m$ as the average clump mass (see Ciotti 2010). 
In axial symmetry, the above terms $h$ and $g$ can be simplified considerably (see Rosenbluth et al. 1956), and to the lowest-order one obtains

$$
\begin{aligned}
& h \simeq 8 \pi\left[\int_{0}^{\nu} \mathrm{d} \nu^{\prime} \frac{\nu^{\prime 2}}{\nu} f_{0}\left(\nu^{\prime}\right)+\int_{\nu}^{\infty} \mathrm{d} \nu^{\prime} \nu^{\prime} f_{0}\left(\nu^{\prime}\right)\right], \\
& g \simeq 4 \pi\left[\nu \int_{0}^{\nu} \mathrm{d} \nu^{\prime} \nu^{\prime 2}\left(1+\frac{\nu^{\prime 2}}{3 \nu^{2}}\right) f_{0}\left(\nu^{\prime}\right)+\int_{\nu}^{\infty} \mathrm{d} \nu^{\prime} \nu^{\prime 3}\left(1+\frac{\nu^{2}}{3 \nu^{\prime 2}}\right) f_{0}\left(\nu^{\prime}\right)\right] ;
\end{aligned}
$$

correspondingly, the Fokker-Planck equation simplifies to

$$
\begin{aligned}
\Gamma_{\star}^{-1}\left(\partial_{t} f\right)_{\star}= & -\frac{1}{\nu^{2}} \partial_{\nu}\left(f \nu^{2} \partial_{\nu} h\right)+\frac{1}{2 \nu^{2}} \partial_{\nu \nu}^{2}\left(f \nu^{2} \partial_{\nu \nu}^{2} g\right)+\frac{1}{2 \nu^{2}} \partial_{\mu \mu}^{2}\left[f \frac{1-\mu^{2}}{\nu} \partial_{\nu} g\right]+ \\
& -\frac{1}{\nu^{2}} \partial_{\nu}\left(f \partial_{\nu} g\right)+\frac{1}{\nu^{2}} \partial_{\mu}\left(f \frac{\mu}{\nu} \partial_{\nu} g\right) .
\end{aligned}
$$

Introducing the relevant integrals

$$
\begin{aligned}
& \mathcal{I}=\int_{0}^{\nu} \mathrm{d} \nu^{\prime} \nu^{\prime 2} f_{0}\left(\nu^{\prime}\right), \\
& \mathcal{J}=\int_{0}^{\nu} \mathrm{d} \nu^{\prime} \nu^{\prime 4} f_{0}\left(\nu^{\prime}\right), \\
& \mathcal{K}=\int_{\nu}^{\infty} \mathrm{d} \nu^{\prime} \nu^{\prime} f_{0}\left(\nu^{\prime}\right),
\end{aligned}
$$

after some manipulation one finds that

$$
\begin{aligned}
& \partial_{\nu} h=-\frac{8 \pi}{\nu^{2}} \mathcal{I} \quad \partial_{\nu \nu}^{2} h=-8 \pi f_{0}, \\
& \partial_{\nu} g=4 \pi\left(\mathcal{I}-\frac{\mathcal{J}}{3 \nu^{2}}+\frac{2}{3} \nu \mathcal{K}\right) \quad \partial_{\nu \nu}^{2} g=\frac{8 \pi}{3}\left(\mathcal{K}+\frac{\mathcal{J}}{\nu^{3}}\right) \quad \partial_{\nu \nu \nu}^{3} g=-8 \pi \frac{\mathcal{J}}{\nu^{4}} .
\end{aligned}
$$

Corresponding to the Legendre expansion for $f$ in Eq. (A2), it is convenient to perform the same for $\left(\partial_{t} f\right)_{\star}$, to read

$$
\left(\partial_{t} f\right)_{\star} \simeq\left(\partial_{t} f_{0}\right)_{\star} P_{0}(\mu)+\left(\partial_{t} f_{2}\right)_{\star} P_{2}(\mu)
$$

Then the Fokker-Planck equation yields

$$
\begin{aligned}
& \Gamma_{\star}^{-1}\left(\partial_{t} f_{0}\right)_{\star} \simeq \frac{4 \pi}{\nu^{2}} \frac{\mathrm{d}}{\mathrm{d} \nu}\left[f_{0} \mathcal{I}+\frac{1}{3 \nu}\left(\mathcal{J}+\nu^{3} \mathcal{K}\right) \frac{\mathrm{d}}{\mathrm{d} \nu} f_{0}\right] \\
& \Gamma_{\star}^{-1}\left(\partial_{t} f_{2}\right)_{\star} \simeq \frac{4 \pi}{\nu^{2}} \frac{\mathrm{d}}{\mathrm{d} \nu}\left[f_{2} \mathcal{I}+\frac{1}{3 \nu}\left(\mathcal{J}+\nu^{3} \mathcal{K}\right) \frac{\mathrm{d}}{\mathrm{d} \nu} f_{2}\right]-\frac{4 \pi}{\nu^{5}}\left(3 \nu^{2} \mathcal{I}+2 \nu^{3} \mathcal{K}-\mathcal{J}\right) f_{2} .
\end{aligned}
$$

Taking now the second-order moments leads to the collision terms

$$
\begin{aligned}
& \left(\partial_{t} \sigma_{r}^{2}\right)_{\star}=8 \pi^{2} \Gamma_{\star} \int_{0}^{\infty} \mathrm{d} \nu\left[\frac{4}{3} \nu(\nu \mathcal{K}-\mathcal{I}) f_{0}-\frac{4}{15}\left(5 \nu \mathcal{I}-\frac{\mathcal{J}}{\nu}\right) f_{2}\right], \\
& \left(\partial_{t} \sigma_{\theta}^{2}\right)_{\star}=8 \pi^{2} \Gamma_{\star} \int_{0}^{\infty} \mathrm{d} \nu\left[\frac{4}{3} \nu(\nu \mathcal{K}-\mathcal{I}) f_{0}+\frac{2}{15}\left(5 \nu \mathcal{I}-\frac{\mathcal{J}}{\nu}\right) f_{2}\right] .
\end{aligned}
$$

With the adopted form of $f$ discussed in $\S \mathrm{A} 1$, we find

$$
\int_{0}^{\infty} \mathrm{d} \nu \nu(\nu \mathcal{K}-\mathcal{I}) f_{0}=0 \quad, \quad \int_{0}^{\infty} \mathrm{d} \nu\left(5 \nu \mathcal{I}-\frac{\mathcal{J}}{\nu}\right) f_{2}=\frac{c_{2} \sigma}{2 \pi^{5 / 2}}
$$

so that the collision terms finally read

$$
\left(\partial_{t} \sigma_{r}^{2}\right)_{\star}=-\frac{64 \sqrt{3 \pi}}{15} G^{2} m \rho \log \Lambda \frac{\sigma_{r}^{2}-\sigma_{\theta}^{2}}{\sigma_{\text {tot }}^{3}},
$$




$$
\left(\partial_{t} \sigma_{\theta}^{2}\right)_{\star}=+\frac{32 \sqrt{3 \pi}}{15} G^{2} m \rho \log \Lambda \frac{\sigma_{r}^{2}-\sigma_{\theta}^{2}}{\sigma_{\mathrm{tot}}^{3}} .
$$

With the effective number of clumps $\mathcal{N} \equiv M / m$ approximately constant, the above collision terms do not break self-similarity, and can be written as

$$
\begin{aligned}
& \left(\Sigma_{r}^{2}\right)_{\star}^{\prime}=-\kappa_{\star} \frac{\mathcal{D} \mathcal{M}}{\Sigma_{\text {tot }}^{3}}\left(\Sigma_{r}^{2}-\Sigma_{\theta}^{2}\right), \\
& \left(\Sigma_{\theta}^{2}\right)_{\star}^{\prime}=+\frac{\kappa_{\star}}{2} \frac{\mathcal{D} \mathcal{M}}{\Sigma_{\text {tot }}^{3}}\left(\Sigma_{r}^{2}-\Sigma_{\theta}^{2}\right) .
\end{aligned}
$$

in terms of the self-similar variables defined in Eqs. (10) of the main text. The strength parameter of dynamical relaxation

$$
\kappa_{\star} \equiv \frac{64 \sqrt{3 \pi}}{405} \frac{\log \mathcal{N}}{\mathcal{N}} \approx 0.5 \frac{\log \mathcal{N}}{\mathcal{N}}
$$

depends mainly on the clumpiness $1 / \mathcal{N}$ of the infalling matter. In Fig. A1 we illustrate $\kappa_{\star}$ as a function of $\mathcal{N}$, and highlight the regimes corresponding to the early fast collapse and to the late slow accretion. In more detail, during the early fast collapse of the halo inner region, a limited effective number of clumps $\mathcal{N} \lesssim 10$ is expected to enforce efficient dynamical relaxation with $\kappa_{\star} \approx 0.1$, while during the late slow accretion dominating the growth of the halo outskirts, many small infalling clumps with $\mathcal{N} \gtrsim 30$ make dynamical relaxation much less efficient with $\kappa_{\star} \lesssim 0.01$. 


\section{Asymptotic Slopes}
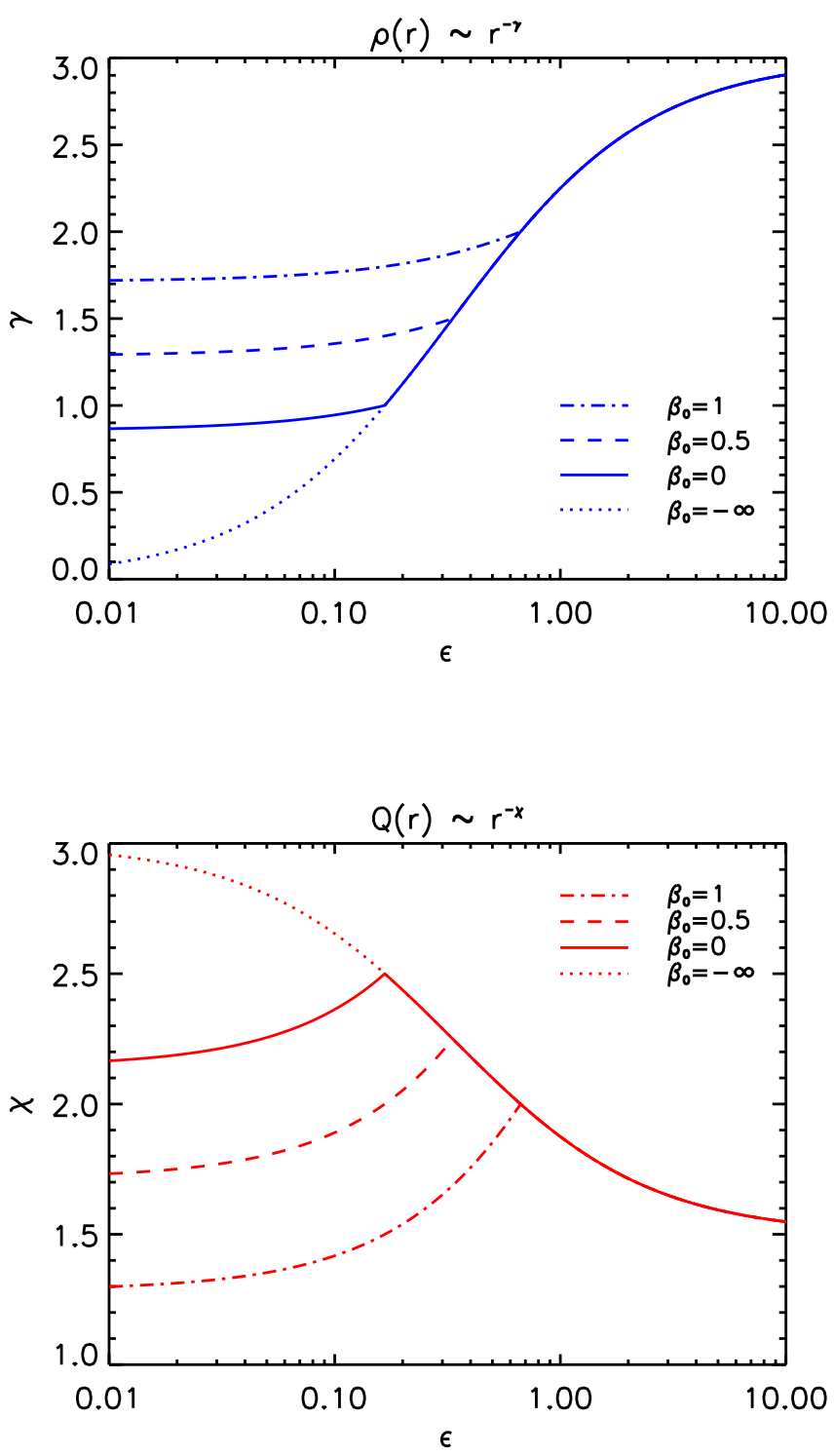

Figure 1. Asymptotic inner slopes of density and (pseudo) phase-space density as a function of $\epsilon$, for different values of the central anisotropy parameter. We recall from $\S 2.1$ that efficient dynamical relaxation enforces $\beta_{0}=0$. 

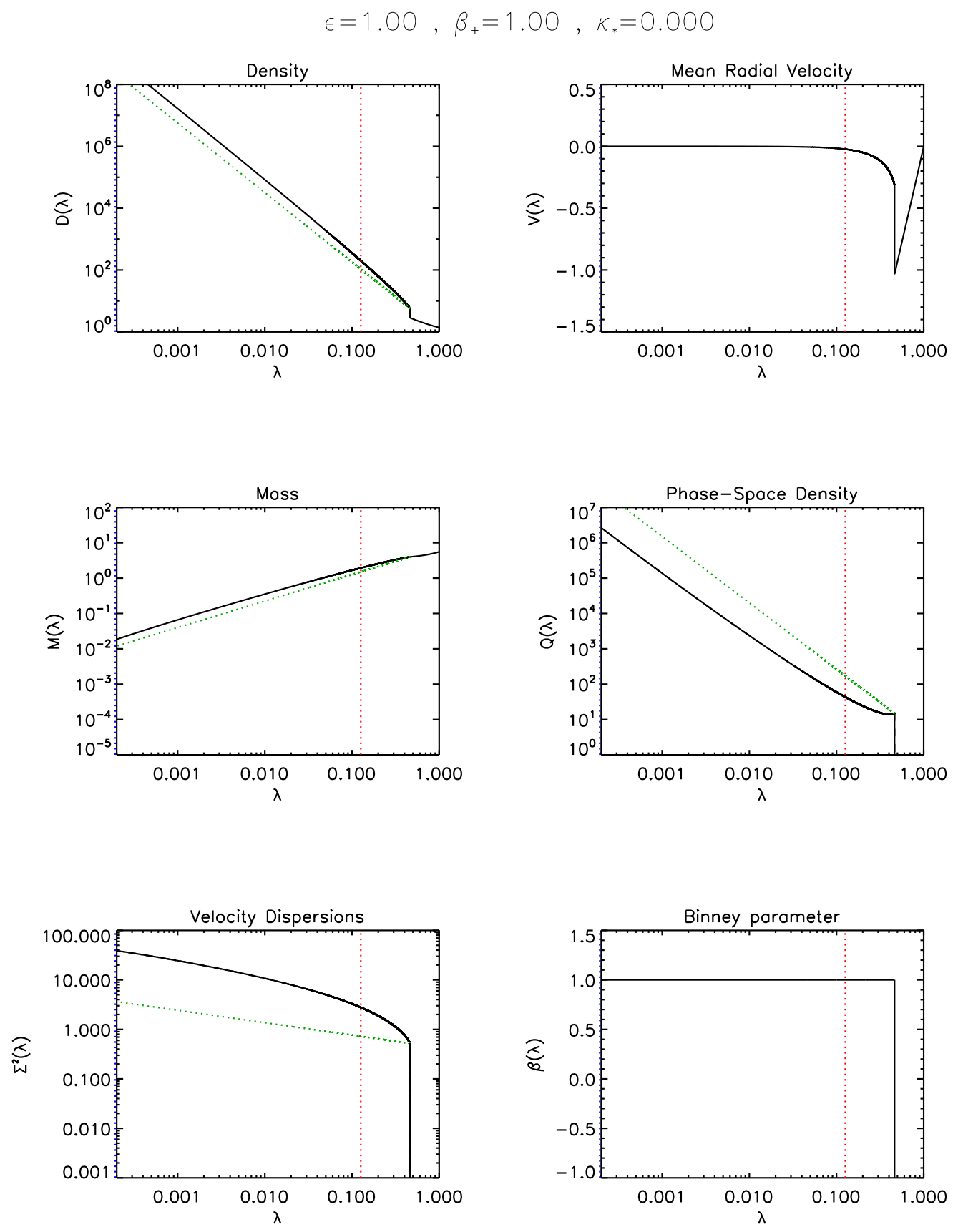

Figure 2. The self-similar solution for $\epsilon=1$ and $\beta_{+}=1$ corresponding to purely radial orbits, in the absence of collisions $\left(\kappa_{\star}=0\right)$; this is just the case considered by Bertschinger (1985). Green dotted lines illlustrate the expected asymptotic behaviors (see $\S 4.2)$. Vertical red lines mark the radius $R_{200}$ where the density amounts to 200 times the background's. Same linestyles are adopted in the following figures. 

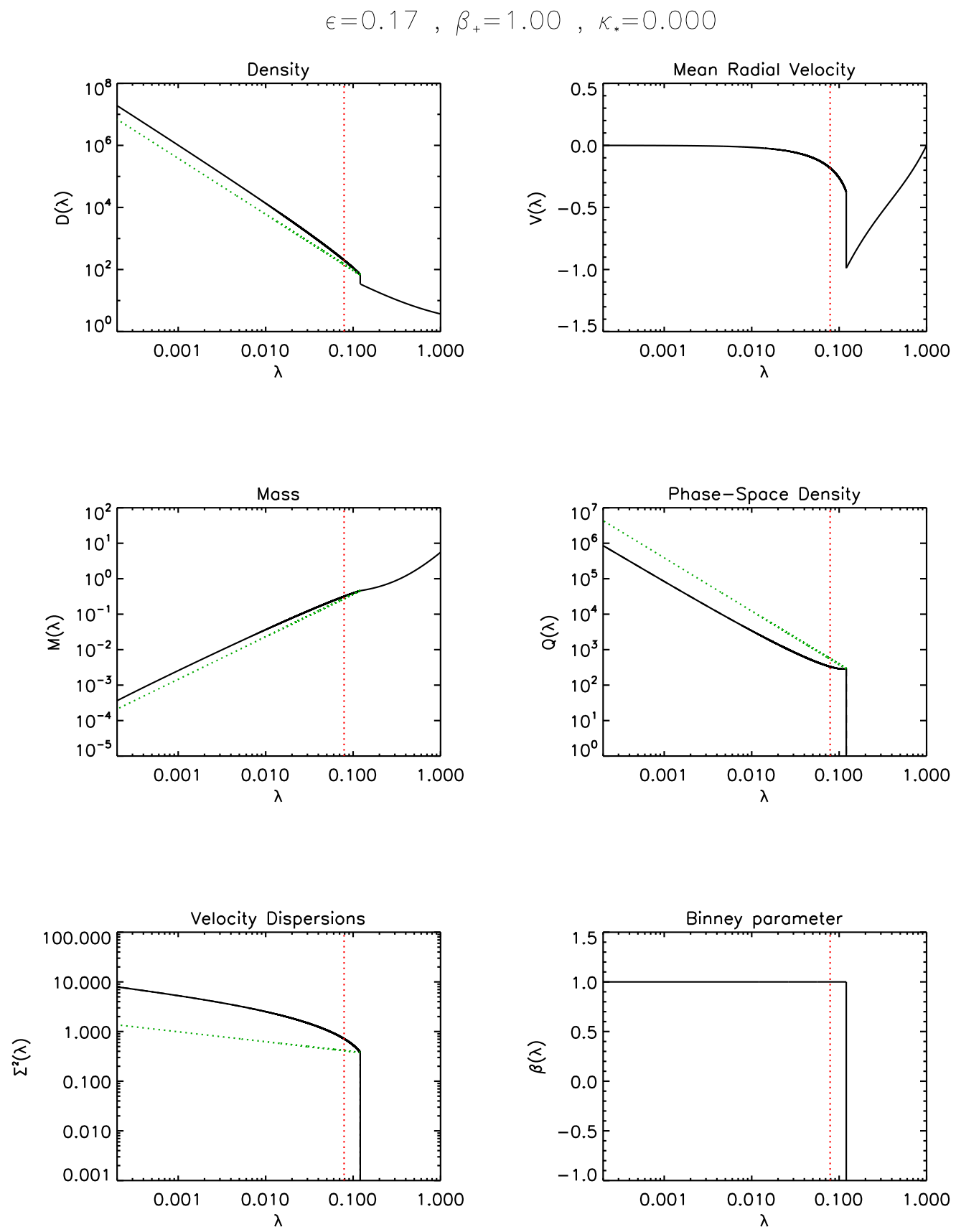

Figure 3. The self-similar solution for $\epsilon=1 / 6$ and $\beta_{+}=1$ corresponding to purely radial orbits, in the absence of collisions $\left(\kappa_{\star}=0\right)$. 

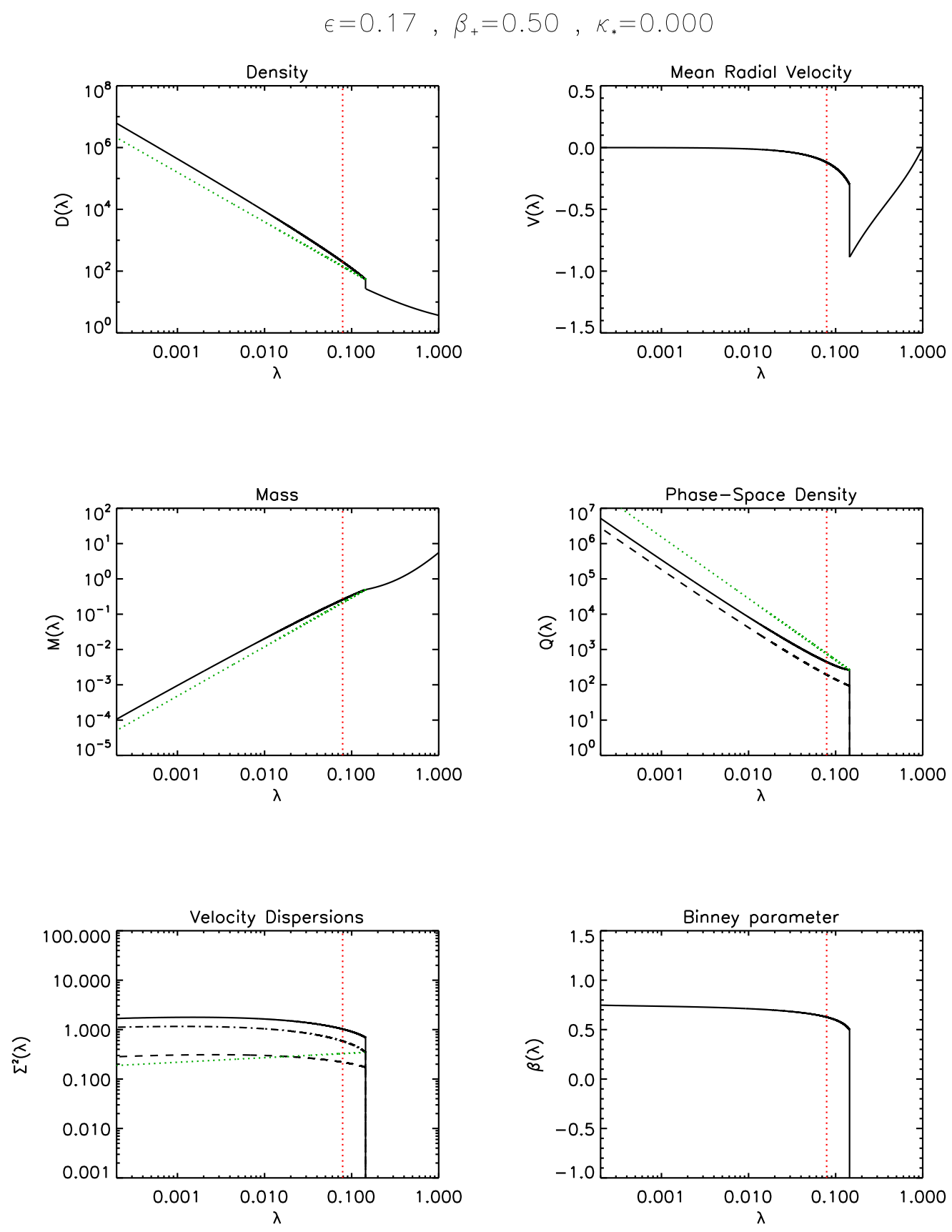

Figure 4. The self-similar solution for $\epsilon=1 / 6$ and $\beta_{+}=0.5$ in the absence of collisions $\kappa_{\star}=0$. In the middle-right panel, solid line refers to $Q(r)$ defined in terms of the total velocity dispersion, while dashed line refers to $Q(r)$ defined in terms of the radial dispersion. In the bottom-left panel, solid line refers to the total velocity dispersion, dot-dashed line to the radial component and dashed line to the tangential component. 

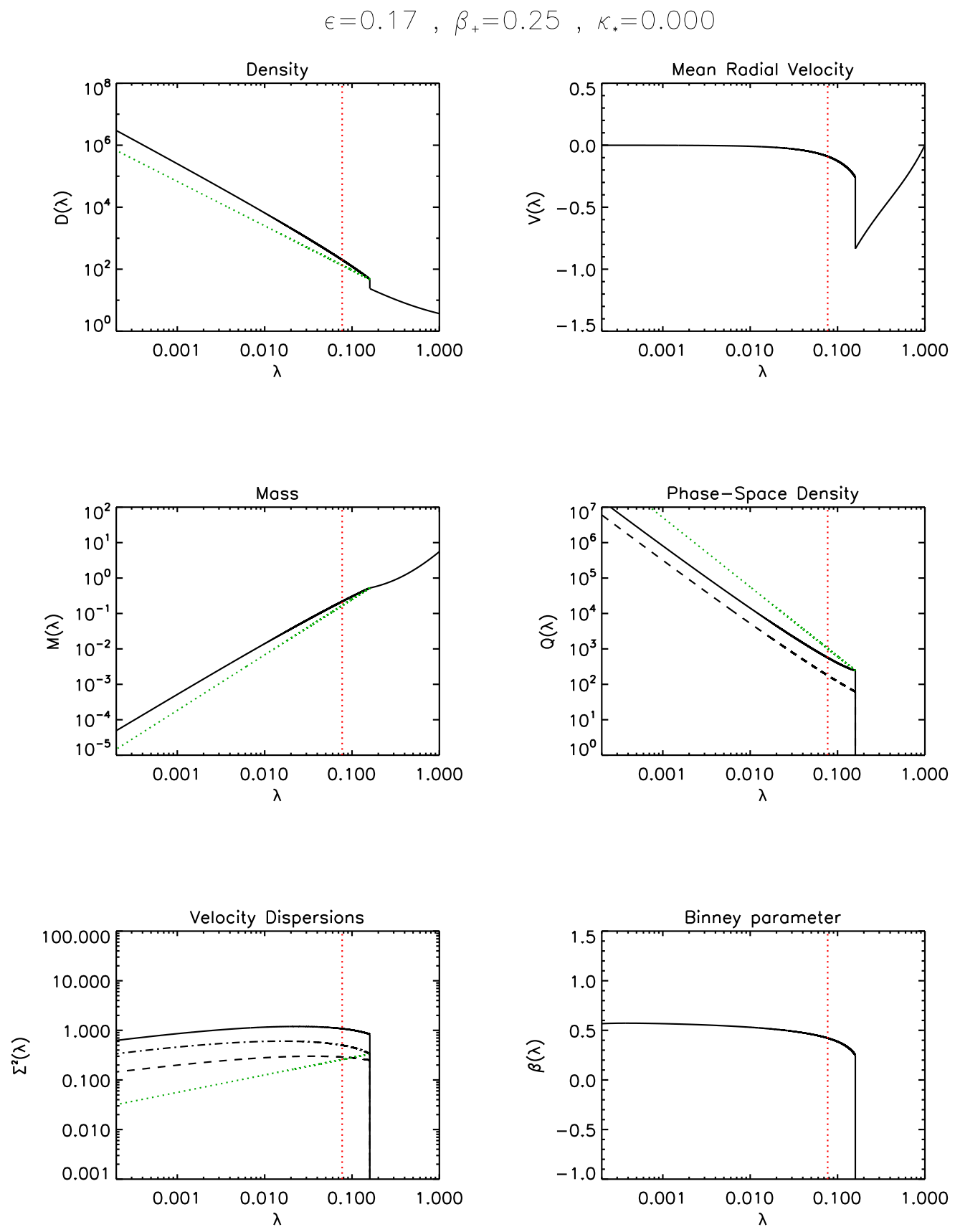

Figure 5. Self-similar solution for $\epsilon=1 / 6$ and $\beta_{+}=0.25$, in the absence of collisions $\kappa_{\star}=0$. 


\section{$\epsilon=0.17, \beta_{+}=0.25, \kappa_{*}=0.010$}
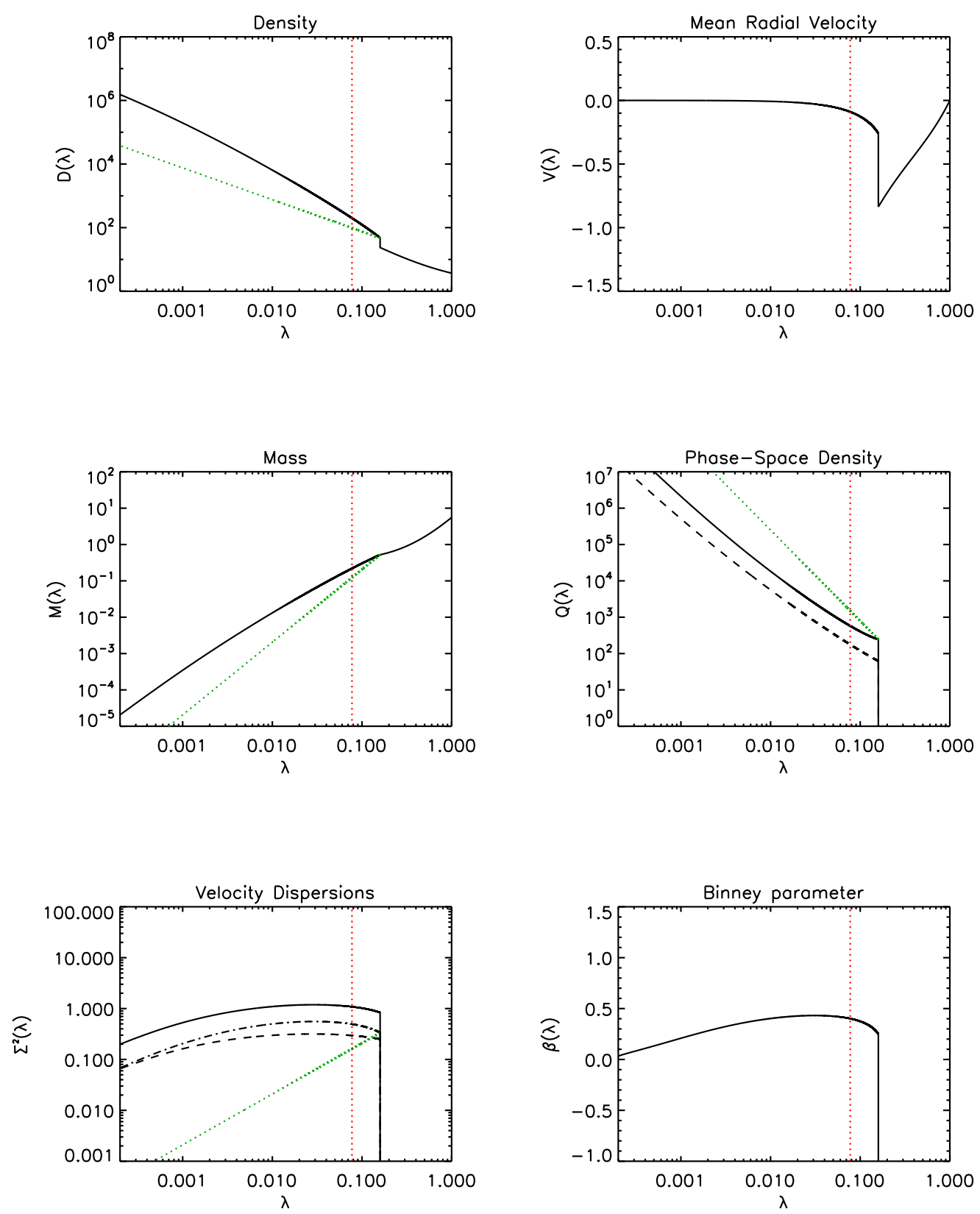

Figure 6. Self-similar solution for $\epsilon=1 / 6$ and $\beta_{+}=0.25$, in presence of collisions with strength parameter $\kappa_{\star}=0.01$. 

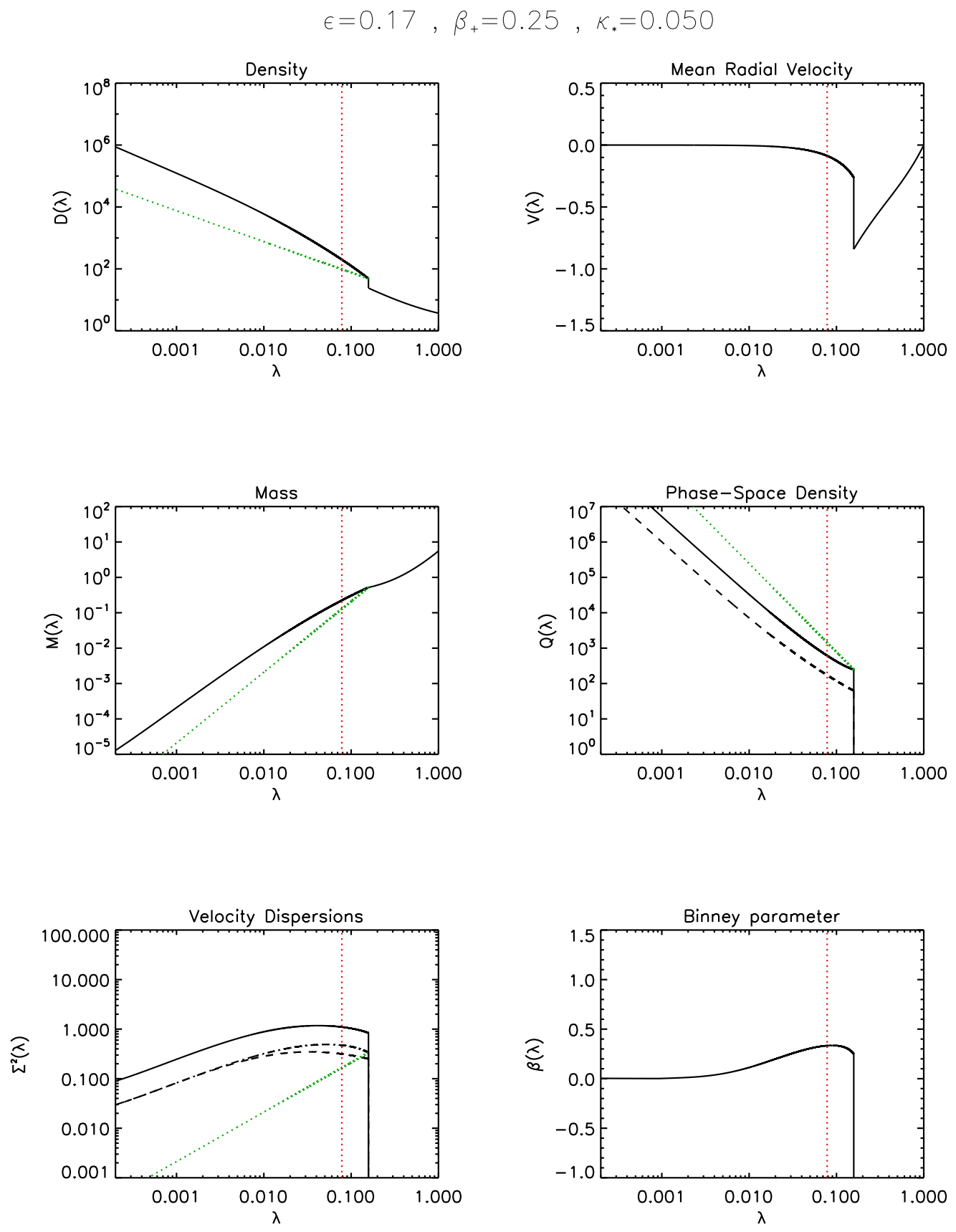

Figure 7. Self-similar solution for $\epsilon=1 / 6$ and $\beta_{+}=0.25$, in presence of collisions with strength parameter $\kappa_{\star}=0.05$. 


\section{$\epsilon=0.17, \beta_{+}=0.25, \kappa_{*}=0.100$}
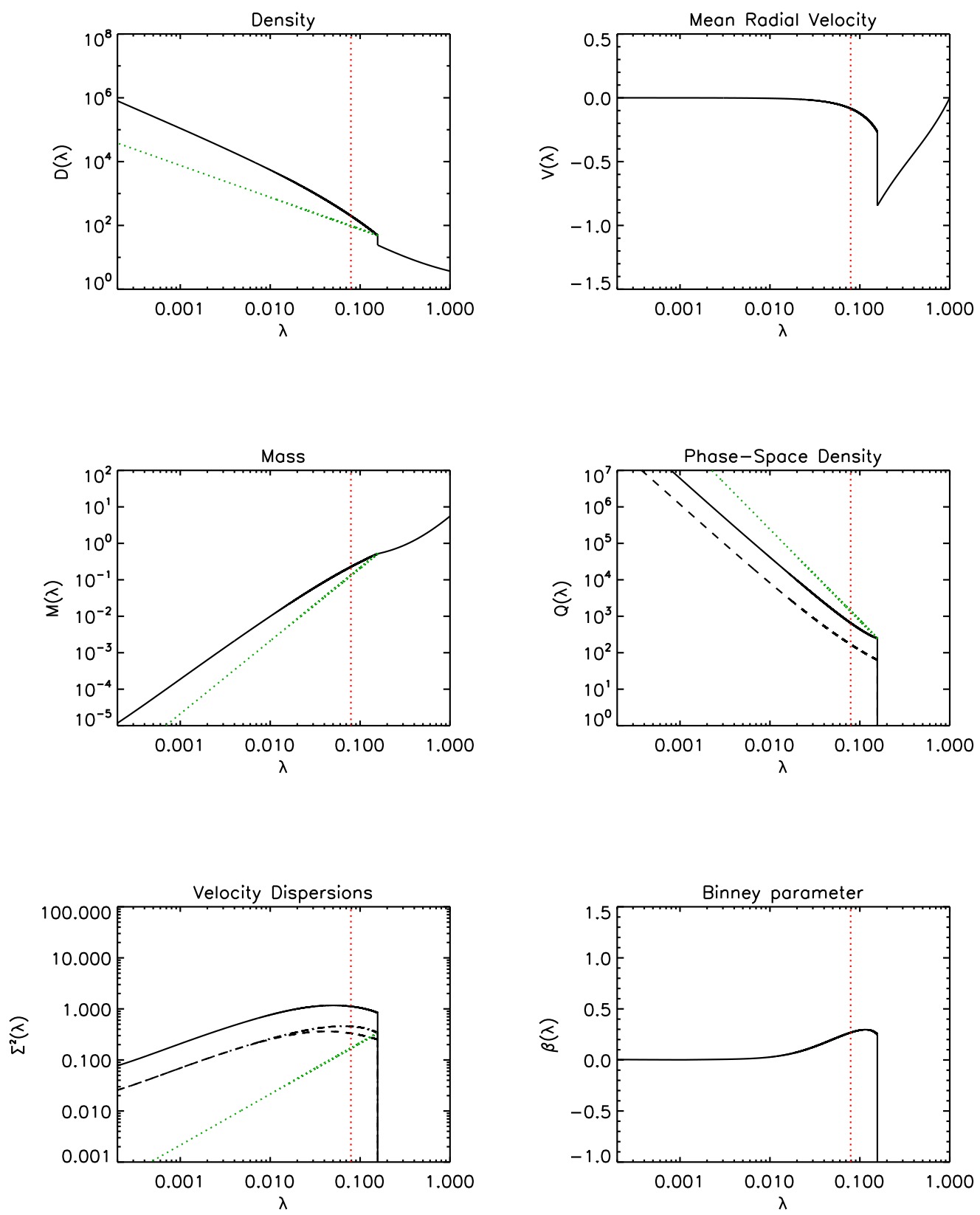

Figure 8. Self-similar solution for $\epsilon=1 / 6$ and $\beta_{+}=0.25$, in presence of collisions with strength $\kappa_{\star}=0.1$. 


\section{$\epsilon=0.13, \beta_{+}=0.25, \kappa_{*}=0.100$}
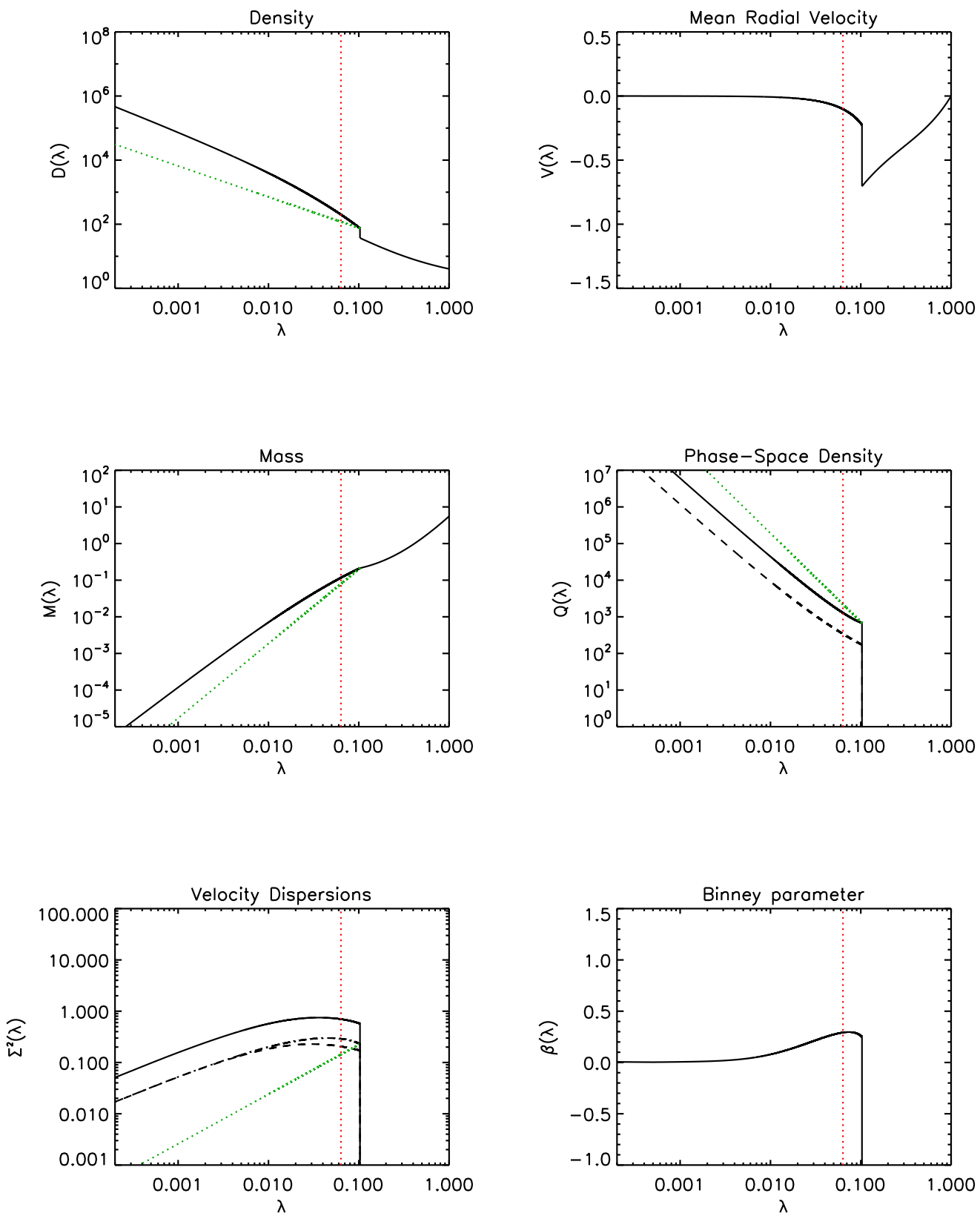

Figure 9. Self-similar solution for $\epsilon=1 / 8$ and $\beta_{+}=0.25$, in presence of collisions with strength $\kappa_{\star}=0.1$. 


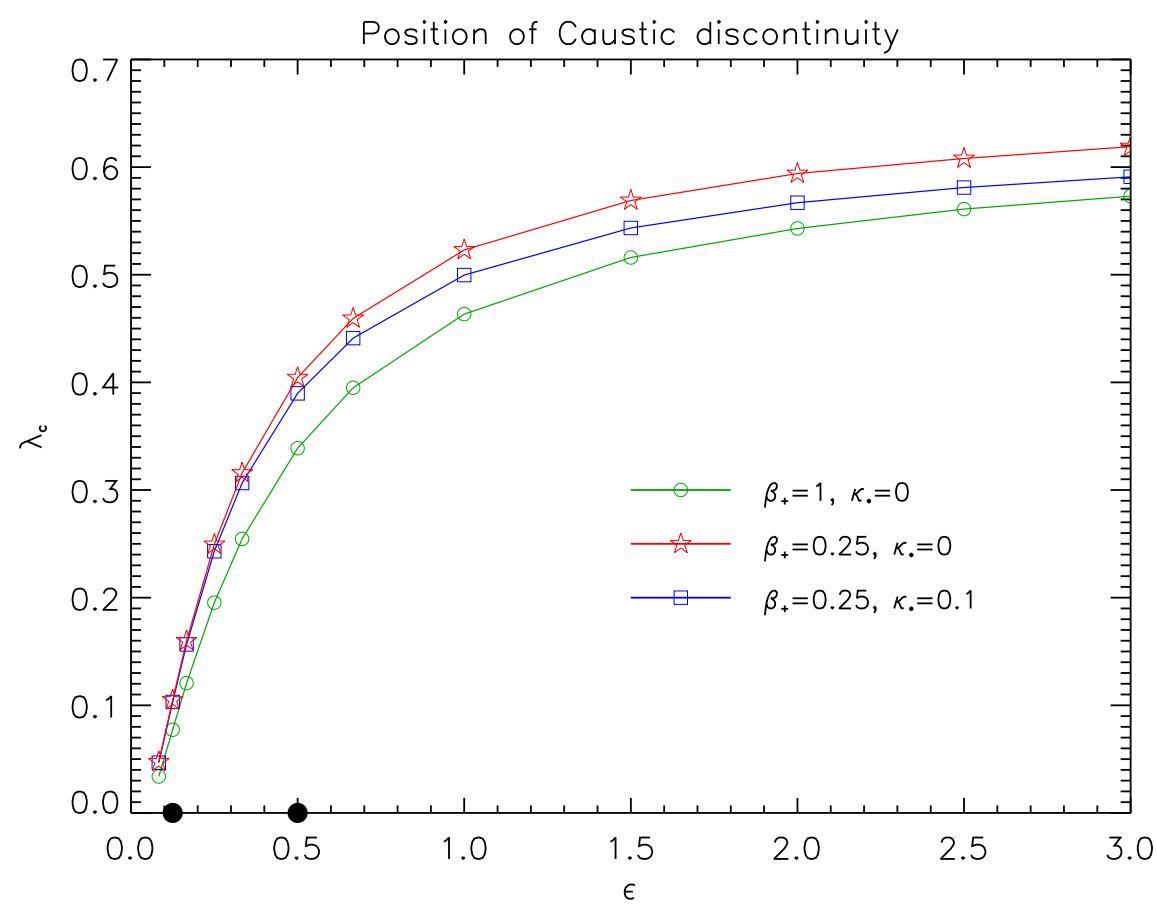

Figure 10. Position of the caustic discontinuity as a function of $\epsilon$, for two values of $\beta_{+}$and $\kappa_{\star}$; these parameters only mildly affect the caustic position at given $\epsilon$. The curves have been computed at discrete values of $\epsilon$, as highlighted with the open symbols; the specific values $\epsilon=1 / 8$ and $1 / 2$ used in the next figures are marked by filled dots. Note that for values $\epsilon \gtrsim 1 / 2$ the caustic position are close to the standard virial radius $R_{200} \approx R_{\mathrm{ta}} / 2$ recalled in $\S 3$. 


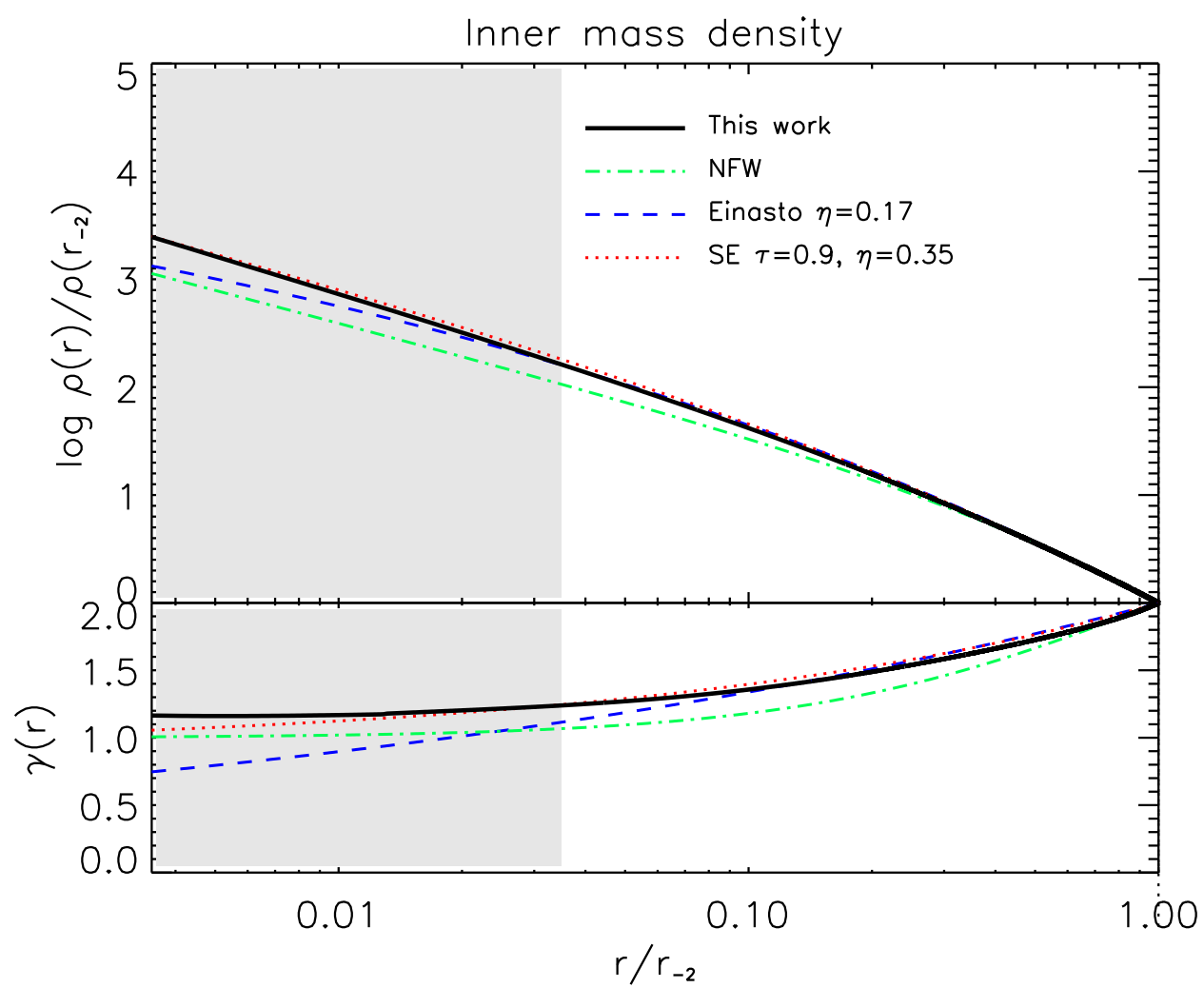

Figure 11. Comparison of the inner self-similar density profile (top) and density slope (bottom) with standard fitting functions to the equilibrium outcomes of numerical simulations. Solid line refers to the self-similar solution with parameters $\epsilon=1 / 8, \beta_{+}=0.25, \kappa_{\star}=0.1$, dot-dashed line refers to the NFW profile, dashed line refers to the Einasto profile with shape parameter $\eta=0.17$, dotted line refers to the Sérsic-Einasto profile with shape parameters $\tau=0.9$ and $\eta=0.35$. The shaded area highlights the radial range not accessible to present numerical simulations. 


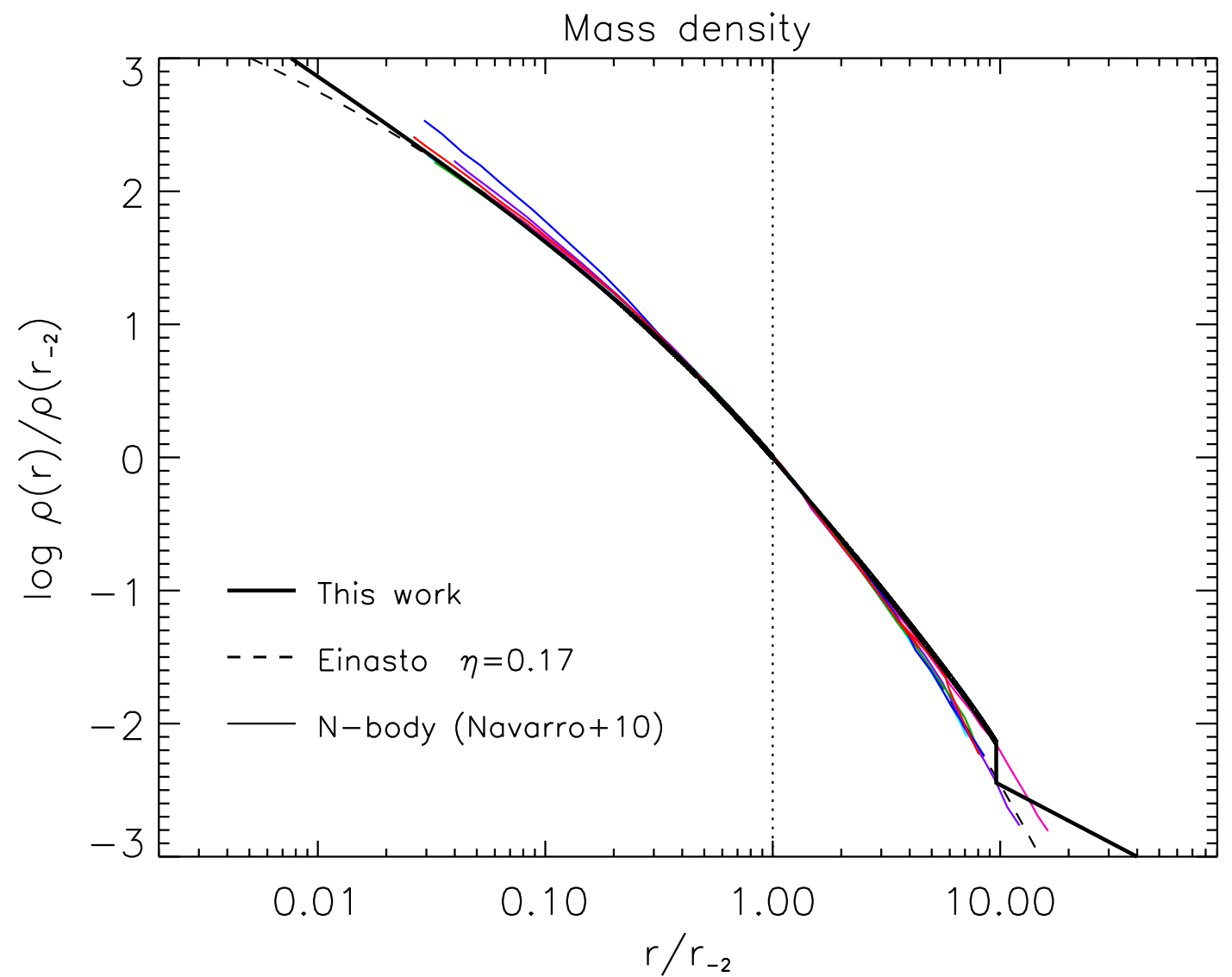

Figure 12. Comparison of the self-similar density profiles (thick solid line) with $\epsilon=1 / 8, \beta_{+}=0.25, \kappa_{\star}=0.1$ (region inward of $r_{-2}$ ) and with $\epsilon=1 / 2, \beta_{+}=0.25, \kappa_{\star}=0.01$ (region outward of $r_{-2}$ ) to the outcomes for six different halos extracted from the Aquarius $N$-body simulation (Navarro et al. 2010; thin colored lines); the standard Einasto profile with $\eta=0.17$ is also illustrated for reference (dashed line). 


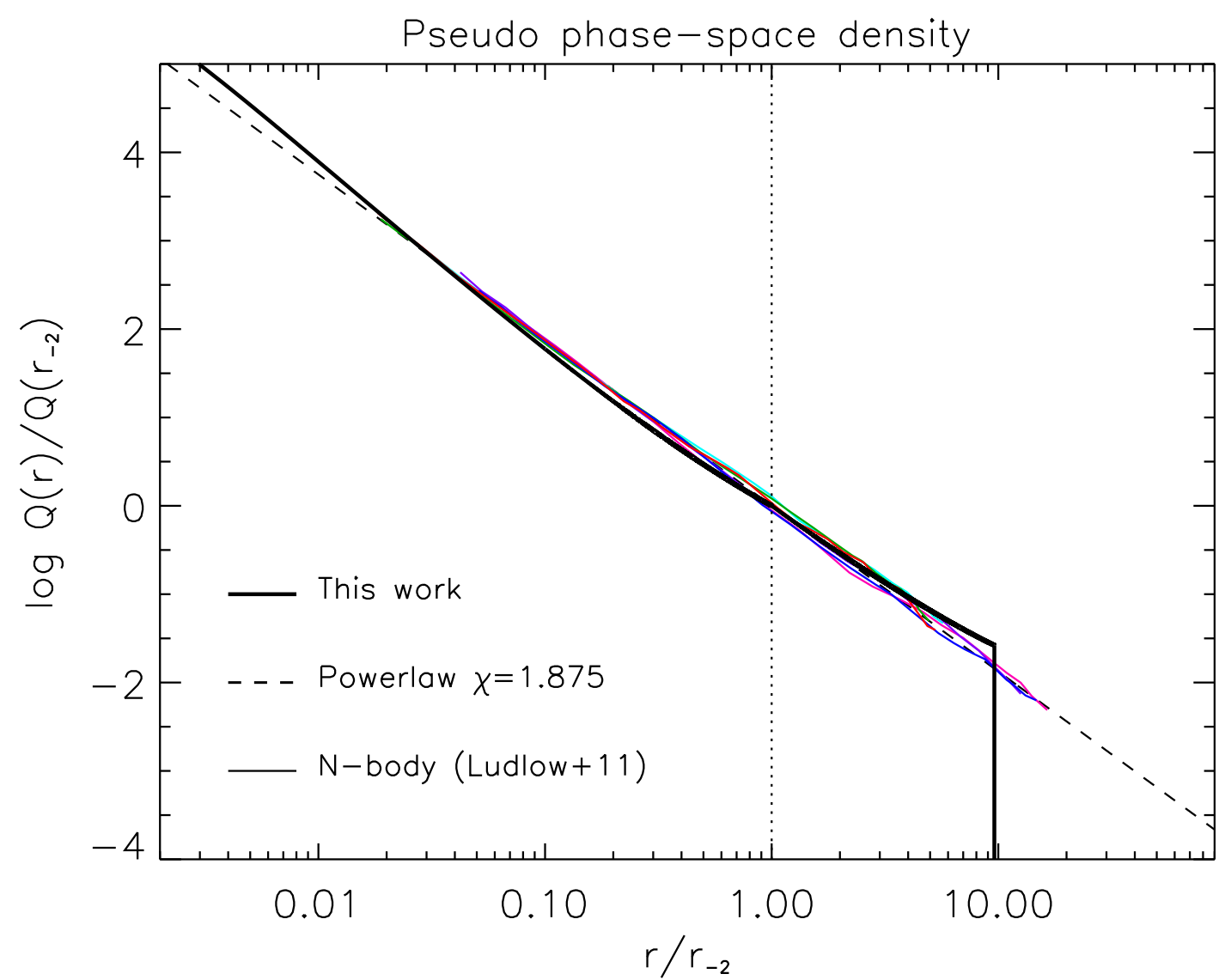

Figure 13. Comparison of the self-similar profiles of pseudo phase-space density (thick solid line) with $\epsilon=1 / 8, \beta_{+}=0.25, \kappa_{\star}=0.1$ (region inward of $r_{-2}$ ) and with $\epsilon=1 / 2, \beta_{+}=0.25, \kappa_{\star}=0.01$ (region outward of $r_{-2}$ ) to the outcomes for six different halos extracted from the Aquarius $N$-body simulation (Navarro et al. 2010; thin colored lines); the powerlaw $Q \propto r^{-\chi}$ with $\chi=1.875$ is also illustrated for reference (dashed line). 


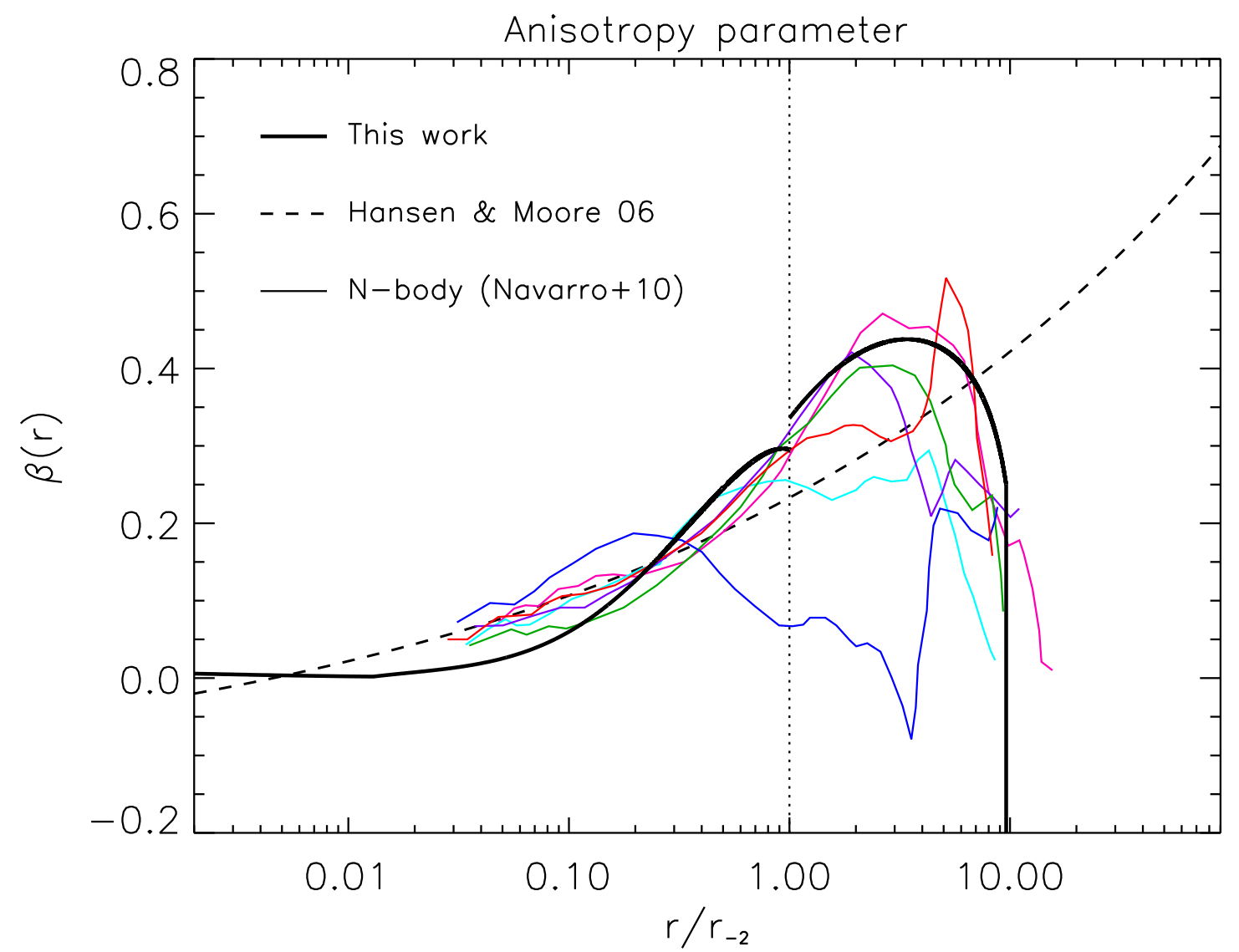

Figure 14. Comparison of the self-similar anisotropy profiles (thick solid line) with $\epsilon=1 / 8, \beta_{+}=0.25, \kappa_{\star}=0.1$ (region inward of $r_{-2}$ ) and with $\epsilon=1 / 2, \beta_{+}=0.25, \kappa_{\star}=0.01$ (region outward of $r_{-2}$ ) to the outcomes for six different halos extracted from the Aquarius $N$-body simulation (Navarro et al. 2010; thin colored lines); the anisotropy profile obtained on combining the Einasto density profile with $\eta=0.17$ and the Hansen \& Moore (2006) $\beta-\gamma$ relation is also illustrated for reference (dashed line). 


\section{HALO HISTORYISTRUCTURE}

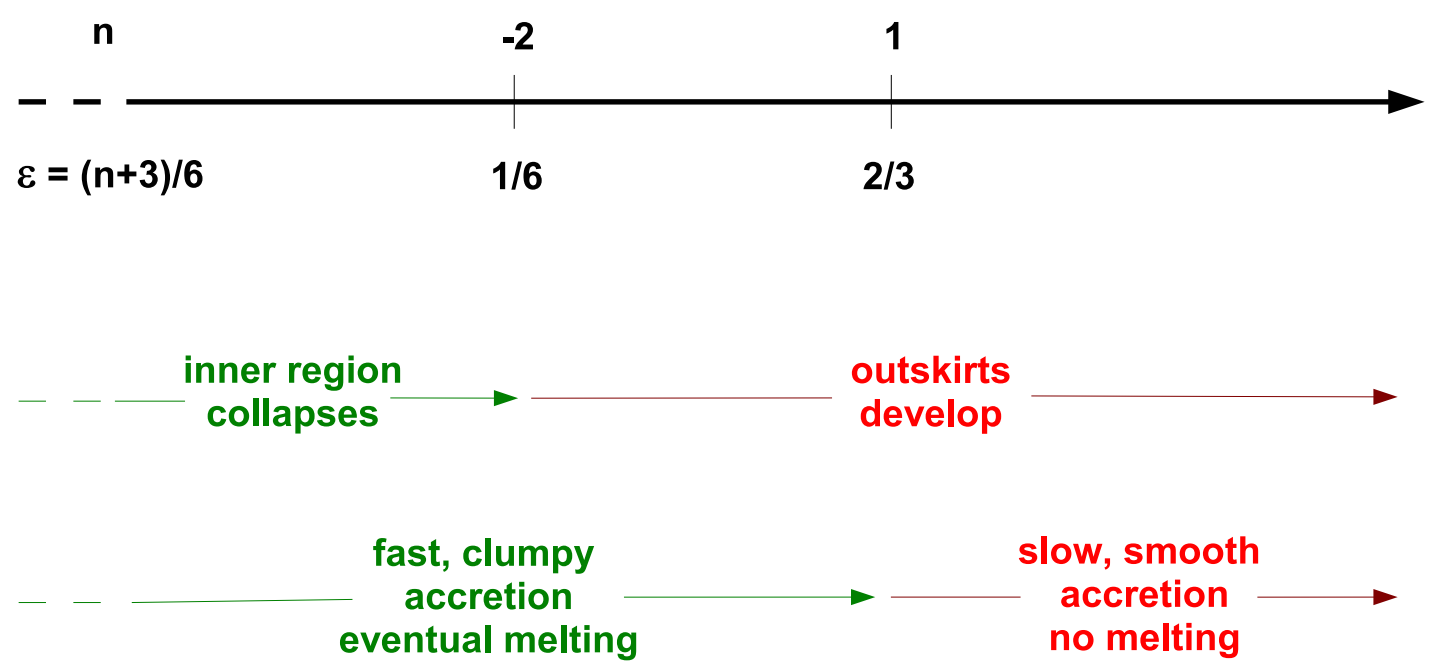

Figure 15. Schematics of the link between the halo structure and its two-stage growth history as it emerges from our self-similar solutions and from numerical simulations (see $\S 5$ for details); note that cosmic time runs from left to right. 


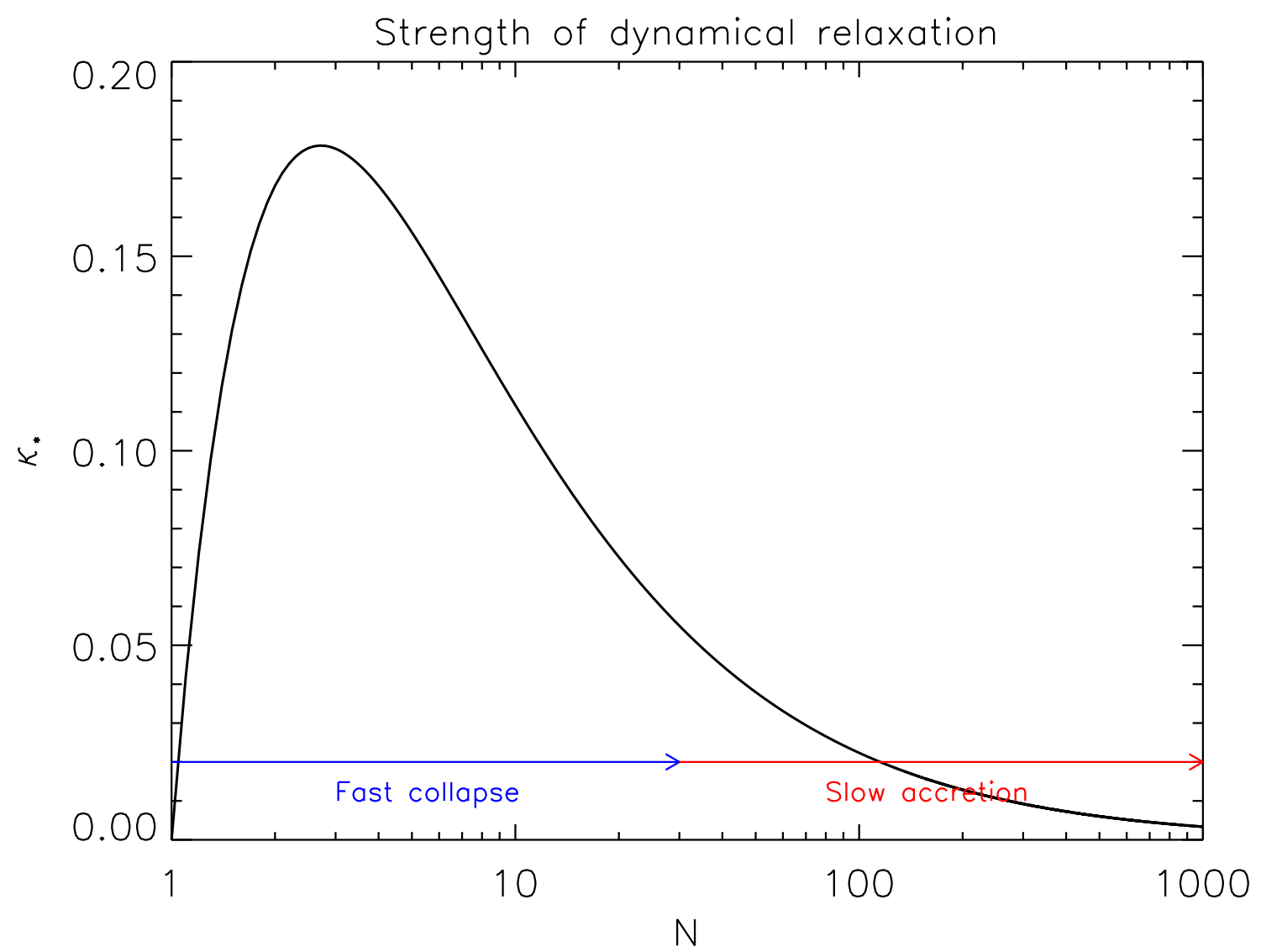

Figure A1. The strength parameter of the dynamical relaxation as a function of the effective number of clumps $\mathcal{N} \equiv M / m$ in the infalling matter, see Appendix A for details. 